\title{
On the Application of Generalized Beta-G Family of Distributions to Prices of Cereals
}

\author{
Rasaki Olawale Olanrewaju \\ Pan African University Institute for Basic Sciences, Technology, and Innovation (PAUSTI), Nairobi, Juja, Kenya \\ Email: rasakiolawale@gmail.com; olanrewaju_rasaq@yahoo.com
}

How to cite this paper: Olanrewaju, R.O. (2021) On the Application of Generalized Beta-G Family of Distributions to Prices of Cereals. Journal of Mathematical Finance, 11, 670-685.

https://doi.org/10.4236/jmf.2021.114036

Received: October 24, 2021

Accepted: November 21, 2021

Published: November 24, 2021

Copyright $\odot 2021$ by author(s) and Scientific Research Publishing Inc. This work is licensed under the Creative Commons Attribution International License (CC BY 4.0).

http://creativecommons.org/licenses/by/4.0/ (c) (i) Open Access

\begin{abstract}
Generalized Beta-G family of distributions proposed has alternative distributions to unbounded distributions for modeling price returns. In contrast to Gaussian and other unbounded distributions that take values from $(-\infty, \infty)$, Generalized Beta-G family of distributions takes values from $[0, \infty)$ so as to properly contain only positive valued observations like that of price returns. In line with this, Nine (9) befitting candidates of the Generalized Beta-G family of distributions were proposed and subjected to monthly prices of cereals. Chen distributional random noise outstripped other candidates of the Generalized Beta-G family of distributions to produce minimum monthly standard deviations of 0.2686 (26.86\%), 0.2572 (25.72\%), 0.2404 (24.40\%), 0.2267 (22.67\%), 0.2257 (22.57\%), 0.2544 (25.44\%), 0.2343 (23.43\%), 0.2391 (23.91\%), $0.2273(22.73 \%)$ and 0.2465 (24.65\%) for prices of Rice, Maize, Sorghum, Millet, G-corn, Cowpea, Groundnut, Beans, Wheat and Cassava respectively. Chen and Loglogistic distributional random noises are the leading candidates among the Generalized Beta-G family of distributions in modelling price returns of the cereals, followed by Fréchet, Weibull and BirnbaumSaunders random noises in order of significant. Lomax and Linear Failure Rate (LFR) are the ineffective random noises in modeling the price returns.
\end{abstract}

\section{Keywords}

Chen, Generalized Beta-G Family of Distributions, Loglogistic, Price, Cereals

\section{Introduction}

Over the past few years, generalization of statistical distribution has attracted much attention. The attention can be classified based on range of values the distribution (s) and subjected matter (s) is/are defined for. When the range of val- 
ues defined for a distribution and the dataset are positively continuous, that is, values taken within $\mathfrak{R}^{+}$, distributions like Life Failure Rate (LFR), Lognormal etc. could be employed so as not to fall the victim of over-parameterization (problem of parsimony). However, when the range of values defined for the distribution and dataset takes range of values from $[0,1]$ or $(0,1)$, distributions like Beta distribution might be the ideal candidate of generalization. In a similar vein, when the range of values for both distribution and dataset takes values on the real number line, that is, $(-\infty, \infty)$, distributions like Gaussian, Gumbel, Student-t, and skew-normal etc. [1].

These distributional generalizations do not only provide robust families of distributions that integrate pliable Probability Density Functions (PDFs) or Probability Mass Functions (PMFs), but also provide ductile functions like, survival \& lifetime analysis functions (both for hazard rate function), reshaping functions (like shape, rate, location, scale, skewness) and quantile function. Each function's candidates have their usefulness, for instance, the location parameter of the reshaping function usually influences the acceptance of a model (that is, the notion of location parameter brings about a better fit), while its absence usually makes a model quite appropriate [2].

Based on studies, generalizations of distributions via some of their mentioned ductile functions do provide bathtub, bathtub-shaped, upside-down bathtub [3]. Among the recently introduced generalized distributions with different appealingness for datasets and users are the new families of distributions. These distributions are Beta exponential-G family introduced by [4], Beta-G family introduced by [5], Generalized Beta-G family by [6], Exponentiated exponential Poisson-G family introduced by [7], Exponentiated-G family introduced by [8], Exponentiated Kumaraswamy-G introduced by [9], Gamma1-G family introduced by [10], Generalized transmuted-G introduced by [11], odd log-logistic-G introduced by [12] among others. Each member of the family of distributions has their own candidates of statistical distributions for statistical modeling or applications to different real life datasets, reliability studies, and importance. Most of these families of distributions are known for modeling lifetime issues, failure rate, time-varying series, price of a commodity, climate change data etc., though simulation studies can also be carried-out so as to estimate probability density function, cumulative distribution function, quantile function, generate random numbers and measures of inference (like Maximum likelihood estimates, Alkaike information criterion, Cramer-von Misses statistic, Anderson-Darling statistic) for each candidate of the distribution that belong to each families of distributions. Each member of the family of the distributions has their own peculiar attributes to applications to datasets. In this research, we shall be narrowing down our scope to Generalized Beta-G family of distributions because of its ability to model failure time events, time remission of bladder cancer patients, climate change agents, flood data, uniform and non-uniform time-varying series like price, stock returns among others. Among the Generalized Beta-G family of distribu- 
tions is Birnbaum-Saunders, Chen, Weibull, Fréchet, F, Life Failure Rate (LFR), Log-logistic, lognormal and Lomax [13].

Among the few applications of the members of the Generalized Beta- $G$ family of distributions to real life events was the application of the extended BirnbaumSaunders distribution (Otherwise known as Marshall-Olkin extended BirnbaumSaunders distribution) to reliability studies and fatigue failure times by [14]. Reference [15] also introduced a modified Burr III distribution called Beta-Burr III distribution and highlighted is importance in modeling problems related to actuarial science and survival analysis. They did not only derive the distribution's docile attributes like the moments (including its moment generating function), reliability, entropies and quantile functions, but also applied it to a survival data of acute myelogeneous Leukaemia of thirty-three (33) patients suffering from the disease.

Reference [16] propounded Beta Gumbel distribution and highlighted its ability to model accelerated life testing problems through earthquakes, flood frequency analysis, rainfall, sea currents, and wind speeds. Reference [17] extended the work of Reference [16] and introduced Beta modified Fréchet distribution called Beta Fréchet (BF) distribution as an extrapolation of Fréchet and Exponentiated Fréchet (EF) distributions. They applied the proposed BF distribution to two sets of data: the uncensored dataset that consist of hundred (100) observations of breaking stress of carbon fibres (in Gba); and used dataset by [18], the dataset that consist of strengths of $1.5 \mathrm{~cm}$ glass fibres measured at the National Physical Laboratory, England. They adopted the Maximum Likelihood method of estimation, and they were able to estimate the four embedded parameters with 95\% confidence level that the BF distribution is an adequate model for modelling the two set of fibres. It is to be noted that Gumbel and Fréchet are two out of the three distributions of the Extreme-Value-Distributions (EVDs). The only notable application of Beta distribution to financial returns was when [19] presented a skewed distribution known as modified Beta distributions and applied it to Standard \& Poor's/International Finance Corporation global daily price indices in United States dollars for South Africa with some inferences made. The statistical properties of the distributions were derived as well as the parameter estimation of the embedded parameters via Maximum Likelihood estimation technique. In light of this, none of the related members or real members of the $\mathrm{Ge}$ neralized Beta-G family of distributions has been applied to stock returns or price indices. The Generalized Beta-G family of distributions is a family of distributions that takes only positive values on the real number line against unbounded distributions that have been used in modeling price indices. The novelty of this work is the first ever application of the Beta-G family of distributions to financial returns of price of commodities, in contrast to its known application to survival analyzes and reliability studies. However, this piece of work will focus on the application of Generalized Beta-G family of distributions to wholesale prices of cereals in Kano state, Nigeria. The wholesale prices of the edible grains to be considered will be from 2007 to 2019 . The members of the 
family of the Generalized Beta-G distributions to be considered are BirnbaumSaunders, Burrxii, Chen, Gamma, Lognormal, Log-Logistic, Lomax, Weibull and Fréchet.

\section{Mathematical Pro-Forma of Price Framework}

Let $p_{0}$ denote the initial price for any commodity/stock returns assuming further that the evolution or time varying for such prices is via the horizon $p=$ one year or $p=$ one month. If the price of such commodity at $\mathrm{p}$ is denoted by $p_{t}$, a random variable, such that,

$$
p=\frac{\ln \left(p_{t}\right)}{\ln \left(p_{0}\right)}=\ln \left(p_{t}\right)-\ln \left(p_{0}\right)
$$

The $\mathrm{p}$ in Equation (1) is also known as growth rate.

Assuming $G$ is a well-defined function on $\mathbb{R}^{+}$with Cumulative Distribution Function (CDF). Let $F$ be another well-defined CDF positioned on $G$ to be the sphere of an increasing function in an enclosed Beta function in the following form:

$$
F(p)=B(G(p, \Omega))
$$

Such that $B:[0,1] \rightarrow[0,1]$ and $\Omega$ being the parameter space of the welldefined $G$ function. The CDF and Probability Density Function (PDF) of the Generalized Beta-G family of distributions can then be defined as:

$$
\begin{gathered}
f(p, \Theta)=\frac{c}{B(a, b)} g(p, \Omega)^{a c-1}\left[1-G^{c}(p, \Omega)\right]^{b-1} \\
F(p, \Theta)=\frac{1}{B(a, b)} \int_{0}^{G^{c}(p, \Theta)} p^{a-1}(1-p)^{b-1} \partial p \\
F^{-1}(r)=G^{-c}\left(I_{r}^{-1}(a, b)\right)
\end{gathered}
$$

for " $r$ " in the range of $g(p, \Omega), 0 \leq r \leq 1 ; \Theta=\left(a, b, c, \Omega^{\mathrm{T}}\right)^{\mathrm{T}}$ is the universal parameter space of the Generalized Beta-G family of distributions with induced shape of $a>0, b>0, c>0 . \Omega$ is the parameter space of the $G(p, \Omega)$ distribution $\ni g(p, \Omega)$ is its pdf. $B(a, b)=\int_{0}^{1} p^{a-1}(1-p)^{b-1} \partial p \quad \& \quad I_{p(a, b)}=\int_{0}^{p} \frac{t^{a-1} \mathrm{e}^{-t} \mathrm{~d} t}{B(a, b)}$ denotes the incomplete beta function ratio. According to [5] and [20], among the few candidates of the Generalized Beta-G family of distributions, that is, the number of independent and identically distributed random variables whose PDF follows $g(p, \Omega)$ are:

Weibull:

$$
g(p, \Omega)=\frac{r}{s}\left(\frac{p-\mu}{s}\right)^{r-1} \exp \left[-\left(\frac{p-\mu}{s}\right)^{r}\right]
$$

For $p>0, p>\mu, \Omega=\{r, s, \mu\}^{\mathrm{T}} \ni \Omega \in \mathfrak{R}^{+} \forall$ shape, rate, and location parameters respectively. 
F:

$$
g(p, \Omega)=B^{-1}\left(\frac{r}{2}, \frac{s}{2}\right)\left(\frac{r}{s}\right)^{\frac{r}{2}}\left(1+r \frac{p-\mu}{s}\right)^{-\left(\frac{r+s}{2}\right)}
$$

$\mathrm{B}$ is stands for the Beta function defined above, for $p>0, p>\mu$, $\Omega=\{r, s, \mu\}^{\mathrm{T}} \ni \Omega \in \mathfrak{R}^{+} \forall$ shape, scale and location parameters respectively.

Linear Failure Rate (LFR):

$$
g(p, \Omega)=(r+s(p-\mu)) \exp \left\{-r p-\frac{(p-\mu)^{2}}{2}\right\}
$$

For $p>0, p>\mu, \Omega=\{r, s, \mu\}^{\mathrm{T}} \ni \Omega \in \mathfrak{R}^{+} \forall$ shape, rate and location parameters respectively.

Chen:

$$
g(p, \Omega)=r s(p-\mu)^{r-1} \exp \left((p-\mu)^{r}\right) \exp \left\{-s\left[\exp \left((p-\mu)^{r}\right)-1\right]\right\}
$$

For $p>0, p>\mu, \Omega=\{r, s, \mu\}^{\mathrm{T}} \ni \Omega \in \mathfrak{R}^{+} \forall$ scale, shape and location parameters respectively.

Birnbaum-Saunders:

$$
g(p, \Omega)=\frac{\sqrt{\frac{s}{p-\mu}}+\sqrt{\frac{p-\mu}{s}}}{2 r(p-\mu)} \phi\left(\frac{\sqrt{\frac{p-\mu}{s}}-\sqrt{\frac{s}{p-\mu}}}{r}\right)
$$

For $p>0, \phi($.$) is the pdf of the standard Gaussian, p>\mu, \Omega=\{s, \mu, r\}^{\mathrm{T}}$ э $\Omega \in \mathfrak{R}^{+} \forall$ scale, location and shape parameters respectively.

Fréchet:

$$
g(p, \Omega)=\frac{r}{s}\left(\frac{p-\mu}{s}\right)^{-r-1} \exp \left\{-\left(\frac{p-\mu}{s}\right)^{-r}\right\}
$$

For $p>0, p>\mu, \Omega=\{r, s, \mu\}^{\mathrm{T}} \ni \Omega \in \mathfrak{R}^{+} \forall$ shape, scale and location parameters respectively.

Log-logistic:

$$
g(p, \Omega)=\frac{r}{s^{r}}(p-\mu)^{r-1}\left[\left(\frac{p-\mu}{s}\right)+1\right]^{-2}
$$

For $p>0, p>\mu, \Omega=\{r, s, \mu\}^{\mathrm{T}} \ni \Omega \in \mathfrak{R}^{+} \forall$ shape, scale and location parameters respectively.

Lomax:

$$
g(p, \Omega)=(\sqrt{2 \pi} s(p-\mu))^{-1} \exp \left[-\frac{1}{2}\left(\frac{\log (p-\mu)-r}{s}\right)^{2}\right]
$$

For $p>0, p>\mu, \Omega=\{r, s, \mu\}^{\mathrm{T}} \ni \Omega \in \mathfrak{R}^{+} \forall$ shape, rate and location parameters respectively.

Log-normal: 


$$
g(p, \Omega)=(\sqrt{2 \pi} s(p-\mu))^{-1} \exp \left[-\frac{1}{2}\left(\frac{\log (p-\mu)-r}{s}\right)^{2}\right]
$$

For $p>0, \pi \approx 3.124, p>\mu, \Omega=\{r, s, \mu\}^{\mathrm{T}}{ }^{\ni} \Omega \in \mathfrak{R}^{+} \forall$ shape, scale and location parameters respectively. It is to be noted that $G(p, \Omega)$ is the CDFs of the pdfs defined above, from Equation (6) to Equation (14). The parameter estimation of the universal parameter space $\Theta=\left(a, b, c, \Omega^{\mathrm{T}}\right)^{\mathrm{T}}$ can be estimated via Maximum Likelihood function of

$$
L(\Theta / P)=\prod_{i=1}^{n}\left(\frac{c}{B(a, b)} g(p, \Omega)^{a c-1}\left[1-G^{c}(p, \Omega)\right]^{b-1}\right)
$$

\section{Numerical Analysis}

The monthly-harmonized wholesale prices (in naira (\#)) of cereals in Kano state, Nigeria from 2007 to 2019 would be subjected to the Generalized Beta-G family of distributions. The cereals include-rice, maize, sorghum, millet, gcorn, cowpea, groundnut, beans, wheat and cassava. The time series dataset was obtained from the Ministry of Agriculture and Natural Resources (MANR), Kano state, Nigeria. The dataset was a monthly uniform time-varying harmonized and regulated price of the edible grains by the ministry (Figure 1).

The median value (that is the black line between the whiskers) for all the cereal prices except for the one of groundnut for the edible grains are more closer to their bottom boxes, with their whiskers shorter on the lower part of their boxes, this suggested an extremely positively skewed distribution (rightly skewed) for all. However, the groundnut possessed the same traits, but not to the extreme like others because the whisker (black line) for the groundnut boxplot was not at the basement of the wall of the plot. In other words, groundnut's

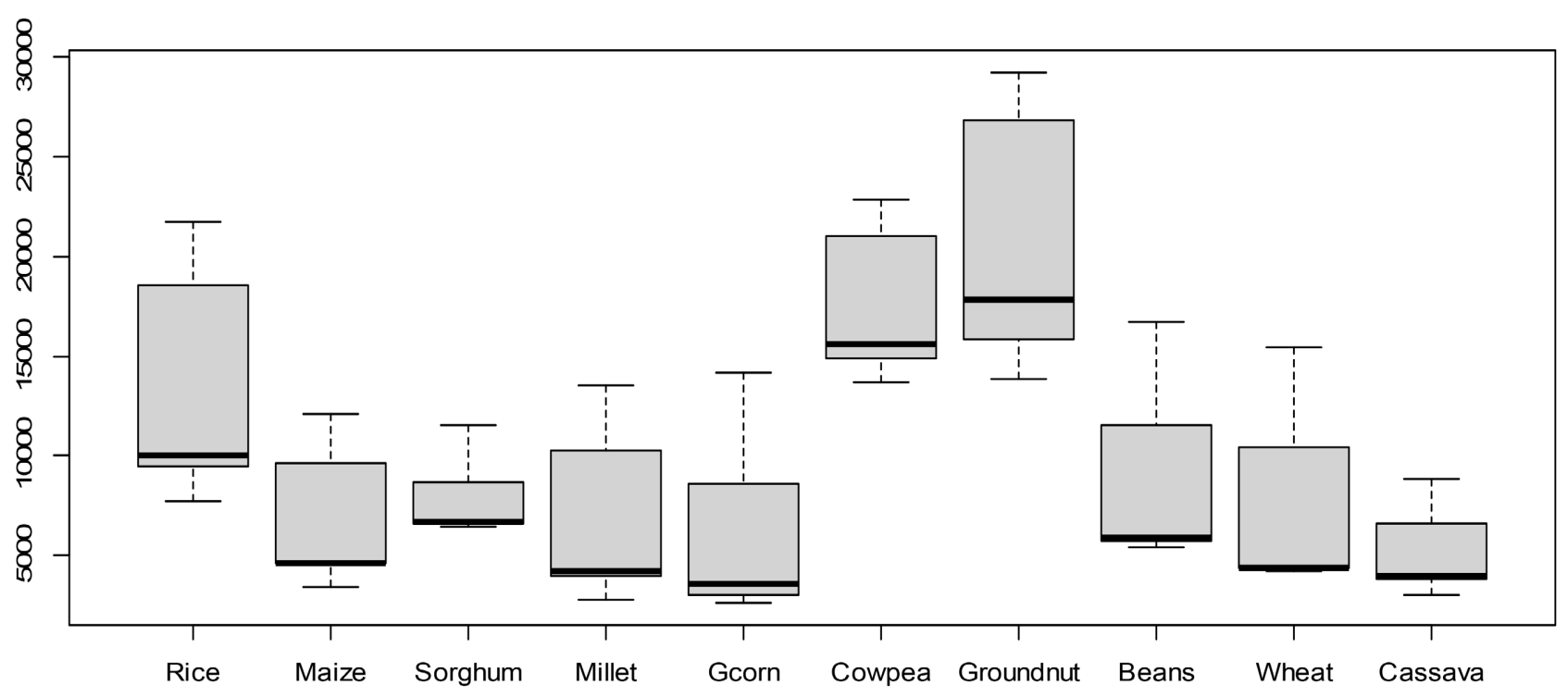

Author's Computation (2021).

Figure 1. Boxplots of the prices of the cereals. 
whisker is in between the median $\left(50^{\text {th }}\right.$ percentile or second quartile) and first quartile $\left(25^{\text {th }}\right.$ percentile), in contrast to others that their whiskers leveled with the first quartile. Overall, it indicated that all the prices of the edible grains are affected by frequent modestly sized deviations that would surely affect estimates if model with Gaussian distribution or unbound distributions.

From Table 1, Chen random noise gave the minimum monthly standard deviation of $0.2686(26.86 \%)$ for the monthly price of rice with moderate magnitude of skewness and kurtosis of 0.1589 and 2.2520 respectively. Interestingly, chen distributional random noise dominated all the other Generalized Beta- $G$ family of distributions in absolving the noise and fluctuations characterized by the prices of cereals to give minimum monthly standard deviations of 0.2572 (25.72\%), 0.2404 (24.04\%), 0.2267 (22.67\%), 0.2257 (22.57\%), 0.2544 (25.44\%), 0.2343 (23.43\%), 0.2391 (23.91\%), 0.2273 (22.73\%), 0.2465 (24.65\%), skewness of 0.2154 , $0.3339,0.1338,0.1773,0.1240,0.3481,0.1277,-0.0152,0.0132$ and kurtosis 2.3266, 2.6471, 2.4149, 2.6258, 2.3426, 2.9886, 2.1858, 2.3668, 2.3813 for prices of Maize, Sorghum, Millet, G-corn, Cowpea, Groundnut, Beans, Wheat and Cassava respectively.

From Table 2, Chen and Weibull are the ideal generalized distributional random noises for rice. They jointly produced the same and smallest reduced error model performance of $\mathrm{AIC}=2973.432$; $\mathrm{CAIC}=2973.996 ; \mathrm{BIC}=2991.731 ; \mathrm{HQIC}=$ 2980.864 with $\hat{\Theta}=(28.4031,8.3039,0.2916,3.7685,1682.6468,7486.7712)$, where $\hat{\Omega}=(3.7685,1682.6468,748.7712)$. From the Anderson-Darling estimate of 9.5843 , that is greater than the critical value of 0.7752 , we fail to accept that the data came from normal distribution. Additionally, since the KolomogorovSmirnov statistic is 0.2701 with its p-value $=0.00011<0.05$ there is no sufficient evidence that the rice price sample came from normal distribution. In addition, chen outmatched other candidates of Generalized Beta-G family of distributions in modelling the price of maize with reduced error performance of AIC = 2812.919; $\mathrm{CAIC}=2813.482 ; \mathrm{BIC}=2831.218 ; \mathrm{HQIC}=2820.351$ with $\hat{\Theta}=(0.1884,5.2514,0.5869,0.1698,0.2694,3440.8126)$, where $\hat{\Omega}=(0.1698,0.2694,3440.8126)$. The Anderson-Darling of $7.1804>0.7752$ shows that the strength of the price of the maize edible grains can be adequately described by the Generalized Beta-G family of distribution. However, since the Kolmogorov-Smirnov statistic is 0.2038 with its p-value $=0.0000<0.05$, it is obvious that price of maize price did not emanate from Gaussian distribution. Fréchet and Loglogistic distributional random noises jointly produced ideal performance for sorghum with $\mathrm{AIC}=2420.474$; $\mathrm{CAIC}=2421.038$; $\mathrm{BIC}=2438.773 ; \mathrm{HQIC}=$ 2427.906, but with different parameters of $\hat{\Theta}=(1.3025,1.0485,3.9379,2.3299,13.5964,128.5248)$ and $\hat{\Theta}=(0.2059,0.1965,0.3054,9.0645,243.2897,6395.0221)$ respectively. Their induced parameters are $\hat{\Omega}=(2.3299,13.5964,128.5248)$ and $\hat{\Omega}=(9.0645,243.2897,6395.0221)$ with Anderson-Darlings' statistic of 12.7501 and $8.8469>0.7752$ and joint Kolomogorov-Smirnovs' p-value $=0.0000<0.05$. Loglogistic and LFR distributional random noises jointly outstripped other 
Table 1. Coefficients of skewness, kurtosis, and standard deviation for the prices of cereals.

\begin{tabular}{|c|c|c|c|c|c|c|c|c|c|}
\hline Rice & Weibull & $\begin{array}{c}\text { Birnabum- } \\
\text { Saunders }\end{array}$ & Chen & $\mathbf{F}$ & Fréchet & LFR & Log-normal & Log-logistic & Lomax \\
\hline $\begin{array}{c}\text { MonthlyStd } \\
\text { Dev }\end{array}$ & 0.9160 & 8.2093 & 0.2686 & 8.1980 & 2.3690 & 3.6767 & 182.3016 & 3.8321 & 1.2433 \\
\hline Skewness & $\begin{array}{c}0.6057 \\
(0.1344)\end{array}$ & $\begin{array}{c}4.5183 \\
(4.6065)\end{array}$ & $\begin{array}{c}0.1589 \\
(0.1620)\end{array}$ & $\begin{array}{c}4.5199 \\
(4.6081)\end{array}$ & $\begin{array}{c}2.4468 \\
(2.4946)\end{array}$ & $\begin{array}{c}2.4654 \\
(3.5612)\end{array}$ & $\begin{array}{c}4.4902 \\
(4.5778)\end{array}$ & $\begin{array}{c}5.3156 \\
(5.4194)\end{array}$ & $\begin{array}{c}9.3941 \\
(9.5775)\end{array}$ \\
\hline Kurtosis & $\begin{array}{c}3.0919 \\
(0.0919)\end{array}$ & $\begin{array}{c}31.9927 \\
(28.9927)\end{array}$ & $\begin{array}{c}2.2520 \\
(-0.7480)\end{array}$ & $\begin{array}{c}25.6861 \\
(22.6861)\end{array}$ & $\begin{array}{l}11.9789 \\
(8.9789)\end{array}$ & $\begin{array}{c}5.6717 \\
(3.9106)\end{array}$ & $\begin{array}{c}27.4719 \\
(24.4719)\end{array}$ & $\begin{array}{c}41.5122 \\
(38.5122)\end{array}$ & $\begin{array}{c}103.6024 \\
(100.6024)\end{array}$ \\
\hline \multicolumn{10}{|l|}{ Maize } \\
\hline $\begin{array}{c}\text { MonthlyStd } \\
\text { Dev }\end{array}$ & 0.9118 & 10.0425 & 0.2572 & 14.2570 & 6.7517 & 0.3099 & 182.3693 & 5.5786 & 2.3576 \\
\hline Skewness & $\begin{array}{c}0.2868 \\
(0.2924)\end{array}$ & $\begin{array}{c}2.8147 \\
(2.8696)\end{array}$ & $\begin{array}{c}0.2154 \\
(0.2197)\end{array}$ & $\begin{array}{c}6.0355 \\
(6.1533)\end{array}$ & $\begin{array}{c}7.2594 \\
(7.4011)\end{array}$ & $\begin{array}{c}0.9326 \\
(0.9508)\end{array}$ & $\begin{array}{c}6.7469 \\
(6.8786)\end{array}$ & $\begin{array}{c}6.8477 \\
(6.8786)\end{array}$ & $\begin{array}{c}4.3167 \\
(3.7543)\end{array}$ \\
\hline Kurtosis & $\begin{array}{c}2.4606 \\
(2.4606)\end{array}$ & $\begin{array}{l}12.8122 \\
(9.8122)\end{array}$ & $\begin{array}{c}2.3266 \\
(-0.673)\end{array}$ & $\begin{array}{c}43.4280 \\
(40.4280)\end{array}$ & $\begin{array}{c}65.0128 \\
(62.0128)\end{array}$ & $\begin{array}{c}3.2869 \\
(0.2869)\end{array}$ & $\begin{array}{c}50.6347 \\
(47.6347)\end{array}$ & $\begin{array}{c}58.9935 \\
(47.6347)\end{array}$ & $\begin{array}{l}34.7172 \\
(7.7256)\end{array}$ \\
\hline \multicolumn{10}{|l|}{ Sorghum } \\
\hline $\begin{array}{c}\text { MonthlyStd } \\
\text { Dev }\end{array}$ & 0.8827 & 9.0662 & 0.2404 & 7.8903 & 3.3496 & 0.2922 & 236.6115 & 2.3323 & 3.4185 \\
\hline Skewness & $\begin{array}{c}0.5483 \\
(0.5590)\end{array}$ & $\begin{array}{c}2.7273 \\
(2.7806)\end{array}$ & $\begin{array}{c}0.3339 \\
(0.3404)\end{array}$ & $\begin{array}{c}5.6829 \\
(5.7939)\end{array}$ & $\begin{array}{c}2.9632 \\
(3.0211)\end{array}$ & $\begin{array}{c}0.9184 \\
(0.9364)\end{array}$ & $\begin{array}{c}9.0870 \\
(9.2644)\end{array}$ & $\begin{array}{c}1.9766 \\
(2.0152)\end{array}$ & $\begin{array}{c}1.7256 \\
(0.7853)\end{array}$ \\
\hline Kurtosis & $\begin{array}{c}2.7043 \\
(-0.2957)\end{array}$ & $\begin{array}{l}11.5328 \\
(8.5328)\end{array}$ & $\begin{array}{c}2.6471 \\
(-0.3529)\end{array}$ & $\begin{array}{c}38.1972 \\
(35.1972)\end{array}$ & $\begin{array}{l}12.9652 \\
(9.9652)\end{array}$ & $\begin{array}{c}2.8979 \\
(-0.1021)\end{array}$ & $\begin{array}{c}95.1809 \\
(95.1809)\end{array}$ & $\begin{array}{c}8.1811 \\
(5.1811)\end{array}$ & $\begin{array}{c}4.3256 \\
(6.4577)\end{array}$ \\
\hline \multicolumn{10}{|l|}{ Millet } \\
\hline $\begin{array}{c}\text { MonthlyStd } \\
\text { Dev }\end{array}$ & 1.0001 & 10.9945 & 0.2267 & 10.6418 & 5.2178 & 0.3194 & 443.5991 & 3.1816 & 2.1778 \\
\hline Skewness & $\begin{array}{c}0.7705 \\
(0.7855)\end{array}$ & $\begin{array}{c}3.4827 \\
(3.5507)\end{array}$ & $\begin{array}{c}0.1338 \\
(0.1364)\end{array}$ & $\begin{array}{c}9.8992 \\
(3.2715)\end{array}$ & $\begin{array}{c}3.2472 \\
(4.2516)\end{array}$ & $\begin{array}{c}1.1351 \\
(1.1573)\end{array}$ & $\begin{array}{c}7.3584 \\
(7.5020)\end{array}$ & $\begin{array}{c}2.8564 \\
(2.9121)\end{array}$ & $\begin{array}{c}10.5606 \\
(10.7668)\end{array}$ \\
\hline Kurtosis & $\begin{array}{c}2.9104 \\
(-0.0896)\end{array}$ & $\begin{array}{c}17.5067 \\
(14.5067)\end{array}$ & $\begin{array}{c}2.4149 \\
(-0.5851)\end{array}$ & $\begin{array}{c}112.6883 \\
(109.6883)\end{array}$ & $\begin{array}{c}7.4357 \\
(12.5467)\end{array}$ & $\begin{array}{c}4.0506 \\
(1.0506)\end{array}$ & $\begin{array}{c}63.0945 \\
(60.0945)\end{array}$ & $\begin{array}{l}12.1612 \\
(9.1612)\end{array}$ & $\begin{array}{c}123.6616 \\
(120.6616)\end{array}$ \\
\hline \multicolumn{10}{|l|}{ G-Corn } \\
\hline $\begin{array}{c}\text { MonthlyStd } \\
\text { Dev }\end{array}$ & 0.8978 & 11.1932 & 0.2257 & 13.8708 & 0.5324 & 0.3192 & 140.8943 & 2.6797 & 0.8967 \\
\hline Skewness & $\begin{array}{c}0.2112 \\
(0.2154)\end{array}$ & $\begin{array}{c}4.6541 \\
(4.7449)\end{array}$ & $\begin{array}{c}0.1773 \\
(0.1807)\end{array}$ & $\begin{array}{c}5.6299 \\
(5.7398)\end{array}$ & $\begin{array}{c}7.2356 \\
(2.3467)\end{array}$ & $\begin{array}{c}0.9829 \\
(1.0021)\end{array}$ & $\begin{array}{c}9.6636 \\
(9.8523)\end{array}$ & $\begin{array}{c}3.5679 \\
(3.6376)\end{array}$ & $\begin{array}{c}7.3981 \\
(7.5425)\end{array}$ \\
\hline Kurtosis & $\begin{array}{c}2.5665 \\
(-0.4335)\end{array}$ & $\begin{array}{c}33.5766 \\
(30.5766)\end{array}$ & $\begin{array}{c}2.6258 \\
(-0.3742)\end{array}$ & $\begin{array}{c}36.4662 \\
(33.4662)\end{array}$ & $\begin{array}{l}23.24352 \\
(56.2461)\end{array}$ & $\begin{array}{c}3.1701 \\
(0.1701)\end{array}$ & $\begin{array}{c}105.0269 \\
(102.0269)\end{array}$ & $\begin{array}{c}19.5677 \\
(16.5677)\end{array}$ & $\begin{array}{c}71.9099 \\
(68.9099)\end{array}$ \\
\hline \multicolumn{10}{|l|}{ Cowpea } \\
\hline $\begin{array}{c}\text { MonthlyStd } \\
\text { Dev }\end{array}$ & 0.8512 & 8.8147 & 0.2544 & 39.7075 & 30.2382 & 0.3027 & 109.7695 & 2.6991 & 0.7178 \\
\hline Skewness & $\begin{array}{c}0.6392 \\
(0.6517)\end{array}$ & $\begin{array}{c}2.6730 \\
(2.7251)\end{array}$ & $\begin{array}{c}0.1240 \\
(0.1264)\end{array}$ & $\begin{array}{c}5.9637 \\
(6.0801)\end{array}$ & $\begin{array}{c}4.2917 \\
(3.2536)\end{array}$ & $\begin{array}{c}0.9304 \\
(0.9486)\end{array}$ & $\begin{array}{c}4.0649 \\
(4.1442)\end{array}$ & $\begin{array}{c}2.8982 \\
(2.9548)\end{array}$ & $\begin{array}{c}5.0566 \\
(5.1553)\end{array}$ \\
\hline
\end{tabular}


Continued

\begin{tabular}{|c|c|c|c|c|c|c|c|c|c|}
\hline Kurtosis & $\begin{array}{c}3.1955 \\
(0.1955)\end{array}$ & $\begin{array}{l}10.9308 \\
(7.9308)\end{array}$ & $\begin{array}{c}2.3426 \\
(-0.6574)\end{array}$ & $\begin{array}{c}42.1433 \\
(39.1433)\end{array}$ & $\begin{array}{c}5.2142 \\
(2.1826)\end{array}$ & $\begin{array}{c}3.5416 \\
(0.5416)\end{array}$ & $\begin{array}{c}20.3783 \\
(17.3783)\end{array}$ & $\begin{array}{c}16.1041 \\
(13.1041)\end{array}$ & $\begin{array}{c}35.5875 \\
(32.5875)\end{array}$ \\
\hline \multicolumn{10}{|l|}{ Groundnut } \\
\hline $\begin{array}{c}\text { MonthlyStd } \\
\text { Dev }\end{array}$ & 0.9267 & 10.3373 & 0.2343 & 17.2021 & 2.7560 & 0.3308 & 438.7740 & 3.2202 & 2.1242 \\
\hline Skewness & $\begin{array}{c}0.4185 \\
(0.4267)\end{array}$ & $\begin{array}{c}2.7455 \\
(2.7990)\end{array}$ & $\begin{array}{c}0.3481 \\
(0.3549)\end{array}$ & $\begin{array}{c}6.1534 \\
(6.2735)\end{array}$ & $\begin{array}{c}3.9229 \\
(3.9995)\end{array}$ & $\begin{array}{c}1.2217 \\
(1.2455)\end{array}$ & $\begin{array}{c}8.8071 \\
(8.9790)\end{array}$ & $\begin{array}{c}3.0847 \\
(3.1449)\end{array}$ & $\begin{array}{c}2.7455 \\
(2.7990)\end{array}$ \\
\hline Kurtosis & $\begin{array}{c}2.9254 \\
(-0.0746)\end{array}$ & $\begin{array}{l}10.8047 \\
(7.8047)\end{array}$ & $\begin{array}{c}2.9886 \\
(-0.0114)\end{array}$ & $\begin{array}{c}43.7856 \\
(40.7856)\end{array}$ & $\begin{array}{c}22.8987 \\
(19.8987)\end{array}$ & $\begin{array}{c}3.7900 \\
(0.7900)\end{array}$ & $\begin{array}{c}81.3375 \\
(78.3375)\end{array}$ & $\begin{array}{c}14.0798 \\
(11.0798)\end{array}$ & $\begin{array}{l}10.8047 \\
(7.8047)\end{array}$ \\
\hline \multicolumn{10}{|l|}{ Beans } \\
\hline $\begin{array}{c}\text { MonthlyStd } \\
\text { Dev }\end{array}$ & 0.8577 & 7.5464 & 0.2391 & 52.9837 & 4.5063 & 0.3239 & 103.6060 & 4.5063 & 1.8774 \\
\hline Skewness & $\begin{array}{c}0.7500 \\
(0.7647)\end{array}$ & $\begin{array}{c}2.4527 \\
(2.5005)\end{array}$ & $\begin{array}{c}0.1277 \\
(0.1302)\end{array}$ & $\begin{array}{c}9.8622 \\
(10.0547)\end{array}$ & $\begin{array}{c}4.2262 \\
(4.3087)\end{array}$ & $\begin{array}{c}1.4355 \\
(1.4635)\end{array}$ & $\begin{array}{c}5.0355 \\
(5.1338)\end{array}$ & $\begin{array}{c}4.2262 \\
(4.3087)\end{array}$ & $\begin{array}{c}7.9103 \\
(8.0647)\end{array}$ \\
\hline Kurtosis & $\begin{array}{c}4.1828 \\
(1.1828)\end{array}$ & $\begin{array}{c}9.5973 \\
(6.5973)\end{array}$ & $\begin{array}{c}2.1858 \\
(-0.8142)\end{array}$ & $\begin{array}{c}108.2008 \\
(105.2008)\end{array}$ & $\begin{array}{c}24.8323 \\
(21.8323)\end{array}$ & $\begin{array}{c}6.3079 \\
(3.3079)\end{array}$ & $\begin{array}{c}33.0210 \\
(30.0210)\end{array}$ & $\begin{array}{c}24.8323 \\
(21.8323)\end{array}$ & $\begin{array}{c}67.4502 \\
(64.4502)\end{array}$ \\
\hline \multicolumn{10}{|l|}{ Wheat } \\
\hline $\begin{array}{c}\text { MonthlyStd } \\
\text { Dev }\end{array}$ & 1.0392 & 9.5383 & 0.2273 & 19.1155 & 3.5880 & 0.3182 & 219.1932 & 3.3448 & 3.3448 \\
\hline Skewness & $\begin{array}{c}0.7992 \\
(0.8148)\end{array}$ & $\begin{array}{c}2.5659 \\
(2.6160)\end{array}$ & $\begin{array}{c}-0.0152 \\
(-0.0155)\end{array}$ & $\begin{array}{c}6.9081 \\
(7.0429)\end{array}$ & $\begin{array}{c}5.0248 \\
(5.1229)\end{array}$ & $\begin{array}{c}1.1461 \\
(1.1685)\end{array}$ & $\begin{array}{c}4.8007 \\
(4.8944)\end{array}$ & $\begin{array}{c}3.2408 \\
(3.3041)\end{array}$ & $\begin{array}{c}3.2408 \\
(3.3041)\end{array}$ \\
\hline Kurtosis & $\begin{array}{c}3.7025 \\
(0.7025)\end{array}$ & $\begin{array}{l}11.0449 \\
(8.0449)\end{array}$ & $\begin{array}{c}2.3668 \\
(-0.6332)\end{array}$ & $\begin{array}{c}55.7194 \\
(52.7194)\end{array}$ & $\begin{array}{c}38.5132 \\
(35.5132)\end{array}$ & $\begin{array}{c}1.1565 \\
(1.1565)\end{array}$ & $\begin{array}{c}27.1498 \\
(24.1498)\end{array}$ & $\begin{array}{c}16.5721 \\
(13.5721)\end{array}$ & $\begin{array}{c}16.5721 \\
(13.5721)\end{array}$ \\
\hline \multicolumn{10}{|l|}{ Cassava } \\
\hline $\begin{array}{c}\text { MonthlyStd } \\
\text { Dev }\end{array}$ & 0.9636 & 12.0795 & 0.2465 & 30.5059 & 12.0795 & 0.3144 & 128.0633 & 2.3483 & 1.1944 \\
\hline Skewness & $\begin{array}{c}0.6517 \\
(0.6644)\end{array}$ & $\begin{array}{c}3.4383 \\
(3.5055)\end{array}$ & $\begin{array}{c}0.0132 \\
(0.0134)\end{array}$ & $\begin{array}{c}8.8221 \\
(8.9943)\end{array}$ & $\begin{array}{c}0.0132 \\
(0.0134)\end{array}$ & $\begin{array}{c}0.9114 \\
(0.9292)\end{array}$ & $\begin{array}{c}7.3775 \\
(7.5215)\end{array}$ & $\begin{array}{c}3.5322 \\
(3.6011)\end{array}$ & $\begin{array}{c}6.0366 \\
(6.1545)\end{array}$ \\
\hline Kurtosis & $\begin{array}{c}2.8589 \\
(-0.1411)\end{array}$ & $\begin{array}{c}16.5949 \\
(13.5949)\end{array}$ & $\begin{array}{c}2.3813 \\
(-0.6187)\end{array}$ & $\begin{array}{c}90.2360 \\
(87.2360)\end{array}$ & $\begin{array}{c}16.5949 \\
(13.5949)\end{array}$ & $\begin{array}{c}3.4208 \\
(0.4208)\end{array}$ & $\begin{array}{c}69.8926 \\
(66.8926)\end{array}$ & $\begin{array}{c}23.0768 \\
(20.0768)\end{array}$ & $\begin{array}{c}43.2602 \\
(40.2602)\end{array}$ \\
\hline
\end{tabular}

Generalized Beta-G family of distributions for price of millet with joint the same and smallest model performance of $\mathrm{AIC}=2840.652$; $\mathrm{CAIC}=2841.216 ; \mathrm{BIC}=$ 2858.951; HQIC $=2848.084$, with the same

$\hat{\Theta}=(0.1744,0.1640,0.6688,7.5087,1400.7276,2779.6405)$, where

$\hat{\Omega}=(7.5087,1400.7276,2779.6405)$, the same Anderson-Darling estimate of $8.7738>0.7752$ and Kolomogorov-Smirnovs' p-value $=0.0000<0.05$. Fréchet distributional random noise produced the ideal performance for price of gcorn with $\mathrm{AIC}=2760.405 ; \mathrm{CAIC}=2241.564 ; \mathrm{BIC}=2778.704 ; \mathrm{HQIC}=2767.956$ with $\hat{\Theta}=(0.1887,0.2012,1.1480,2.8519,712.9693,2598.7736)$, such that the induced parameter is $\hat{\Omega}=(2.8519,712.9693,2598.7736)$. Its Anderson-Darling estimate 
Table 2. Model adequacy for the generalized Beta-G family of distributions with the prices of cereals.

\begin{tabular}{|c|c|c|c|c|c|c|c|c|c|c|c|c|c|c|c|c|c|c|c|c|c|}
\hline$\tilde{y}$ & 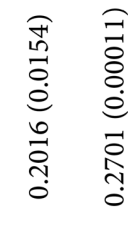 & 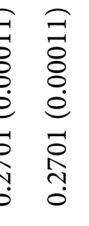 & 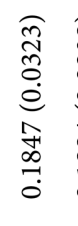 & 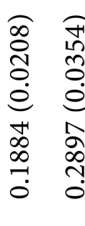 & $\begin{array}{l}\widehat{\overrightarrow{0}} \\
\tilde{0} \\
0 \\
0 \\
0 \\
\infty \\
0 \\
0\end{array}$ & $\begin{array}{l}\widehat{\overrightarrow{0}} \\
00 \\
0 \\
0 \\
0 \\
\infty \\
0 \\
0 \\
0\end{array}$ & 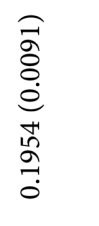 & $\begin{array}{l}\widehat{\sigma} \\
\vdots \\
0 \\
0 \\
0 \\
0 \\
0 \\
0 \\
0\end{array}$ & 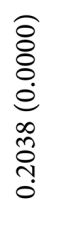 & 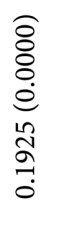 & 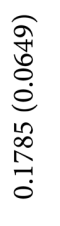 & 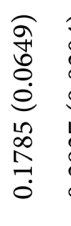 & 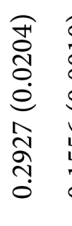 & $\begin{array}{l}0 \\
\stackrel{0}{0} \\
0 \\
0 \\
0 \\
0 \\
0 \\
0 \\
0 \\
0 \\
0\end{array}$ & 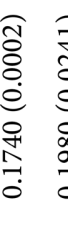 & & & 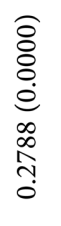 & & 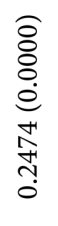 & 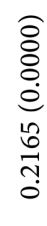 \\
\hline & 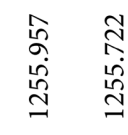 & 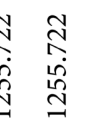 & 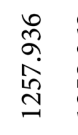 & 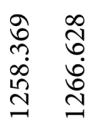 & 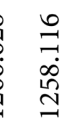 & 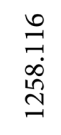 & $\begin{array}{l}\stackrel{\text { If }}{\mathrm{d}} \\
\text { d } \\
\mathbb{1}\end{array}$ & 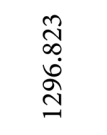 & 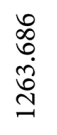 & 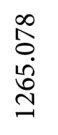 & 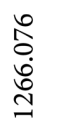 & 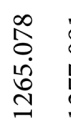 & $\begin{array}{l}\vec{\partial} \\
\stackrel{i}{A} \\
\text { }\end{array}$ & 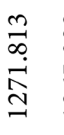 & & 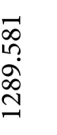 & & 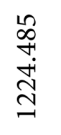 & & $\begin{array}{l}\stackrel{m}{7} \\
\stackrel{0}{\Xi} \\
\Xi\end{array}$ & \\
\hline \& & 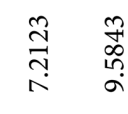 & 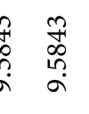 & 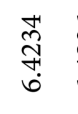 & 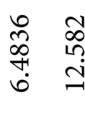 & 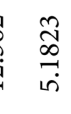 & $\begin{array}{l}\tilde{A} \\
\stackrel{\infty}{1} \\
\dot{10}\end{array}$ & 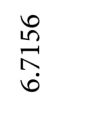 & 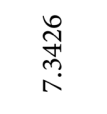 & 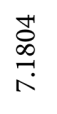 & 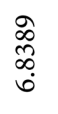 & 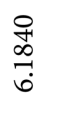 & $\begin{array}{c}\infty \\
\infty \\
\infty \\
\infty \\
\dot{0}\end{array}$ & 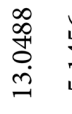 & 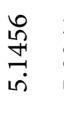 & 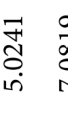 & 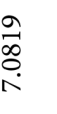 & & 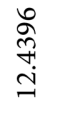 & & $\begin{array}{l}\vec{\vdots} \\
\stackrel{n}{i} \\
\text { i }\end{array}$ & \\
\hline$\sum_{0}$ & 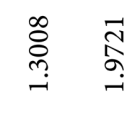 & 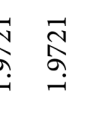 & $\underset{ت}{\vec{G}}$ & 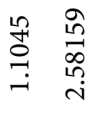 & $\left\{\begin{array}{l}0 \\
h \\
\text { h } \\
0 \\
0\end{array}\right.$ & $\begin{array}{l}\text { o } \\
\text { م⿱ } \\
0 \\
0\end{array}$ & 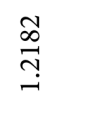 & 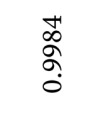 & 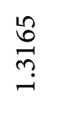 & $\underset{\stackrel{\vec{n}}{\rightarrow}}{\stackrel{\vec{n}}{\longrightarrow}}$ & $\begin{array}{l}\stackrel{\partial}{\vec{े}} \\
\stackrel{-}{-}\end{array}$ & $\underset{\stackrel{n}{n}}{\stackrel{\sim}{\rightarrow}}$ & 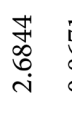 & $\begin{array}{l}\mathbb{E} \\
\text { ¿ } \\
0 \\
0\end{array}$ & 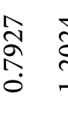 & ¿্ঠ & & 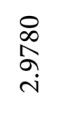 & & $\begin{array}{l}\text { ఫે } \\
\text { ఫे } \\
\text { i }\end{array}$ & \\
\hline & 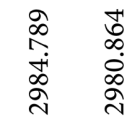 & 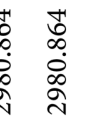 & $\begin{array}{l}\underset{N}{*} \\
\stackrel{+}{+} \\
\stackrel{N}{\Delta}\end{array}$ & 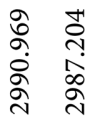 & 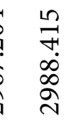 & 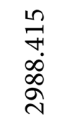 & 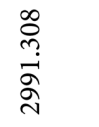 & $\begin{array}{l}\text { aे } \\
0 \\
0 \\
0 \\
0 \\
0\end{array}$ & 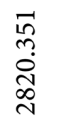 & $\begin{array}{l}\infty \\
\infty \\
\stackrel{\infty}{\sim} \\
\infty \\
\infty \\
\sim\end{array}$ & 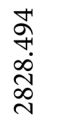 & 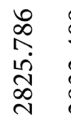 & 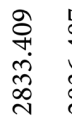 & & 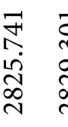 & 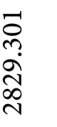 & & $\begin{array}{l}\infty \\
\stackrel{\infty}{\alpha} \\
\stackrel{\hat{N}}{\hat{n}} \\
\hat{n}\end{array}$ & & 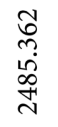 & \\
\hline$\stackrel{\cup}{,}$ & 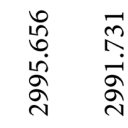 & 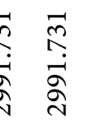 & $\begin{array}{l}\infty \\
i n \\
\kappa \\
\alpha \\
\alpha\end{array}$ & 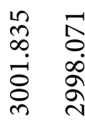 & 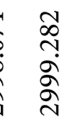 & 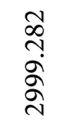 & 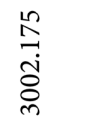 & 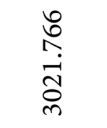 & $\begin{array}{l}\stackrel{\infty}{\sim} \\
\underset{\vec{\sim}}{\infty} \\
\stackrel{\infty}{\sim}\end{array}$ & 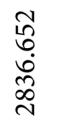 & 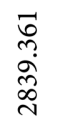 & 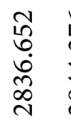 & 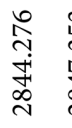 & & 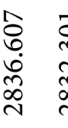 & 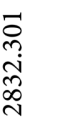 & & $\begin{array}{l}\stackrel{0}{0} \\
\infty \\
\infty \\
0 \\
0 \\
0 \\
m\end{array}$ & m & 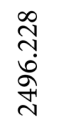 & I \\
\hline & 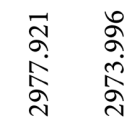 & 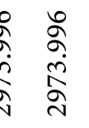 & 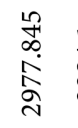 & 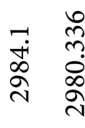 & 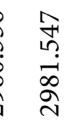 & 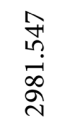 & 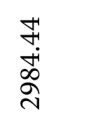 & 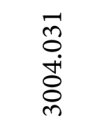 & 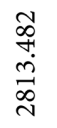 & $\begin{array}{l}\bar{a} \\
\vec{\infty} \\
\infty \\
\stackrel{\infty}{\sim}\end{array}$ & 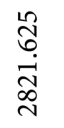 & $\begin{array}{l}\bar{a} \\
\vec{\infty} \\
\infty \\
\stackrel{\infty}{\infty}\end{array}$ & 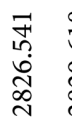 & 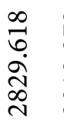 & 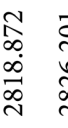 & & & $\underset{\hat{a}}{\stackrel{a}{n}}$ & 1 & 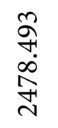 & \\
\hline$=$ & 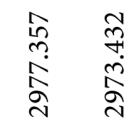 & 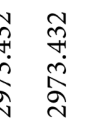 & $\begin{array}{l}\vec{\infty} \\
\stackrel{+}{1} \\
\stackrel{N}{\hat{N}}\end{array}$ & 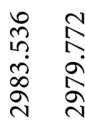 & 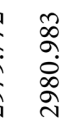 & 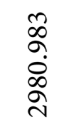 & 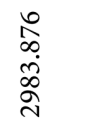 & 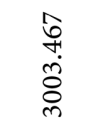 & 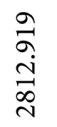 & $\begin{array}{l}\hat{n} \\
\substack{n \\
\infty \\
\infty \\
\infty \\
\infty \\
\sim}\end{array}$ & 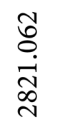 & 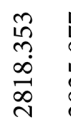 & 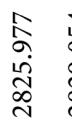 & 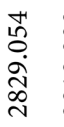 & 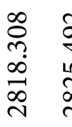 & 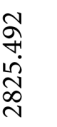 & & $\begin{array}{l}\text { o } \\
0 \\
0 \\
0 \\
0 \\
m \\
m\end{array}$ & 夏 & 空 & \\
\hline$₹$ & 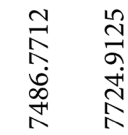 & 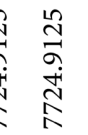 & 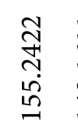 & 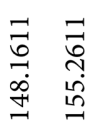 & 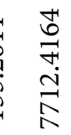 & $\begin{array}{l}\vec{J} \\
\overrightarrow{7} \\
\mathbb{i} \\
\mathbb{N}\end{array}$ & 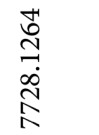 & 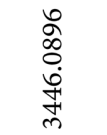 & 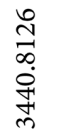 & 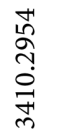 & $\begin{array}{l}\text { na } \\
\overrightarrow{0} \\
\stackrel{0}{0} \\
0 \\
0\end{array}$ & 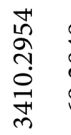 & 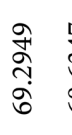 & $\begin{array}{l}\text { fy } \\
\text { o. } \\
\text { o. } \\
0\end{array}$ & 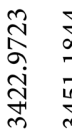 & 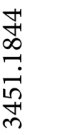 & & 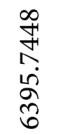 & C & 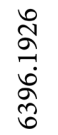 & $\stackrel{m}{a}$ \\
\hline 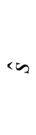 & $\begin{array}{ll}\infty & 0 \\
0 & 0 \\
0 & 0 \\
i & 0 \\
0 & \infty \\
0 & 0\end{array}$ & 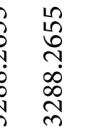 & $\underset{\substack{\infty \\
m}}{\infty}$ & 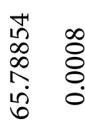 & 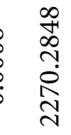 & 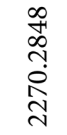 & 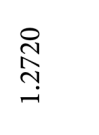 & 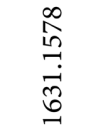 & 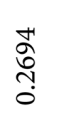 & 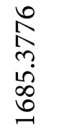 & $\begin{array}{l}\tilde{\sigma} \\
\hat{o} \\
\infty \\
\infty\end{array}$ & 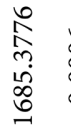 & $\begin{array}{l}0 \\
\vdots \\
\vdots\end{array}$ & $\begin{array}{l}\frac{m}{F} \\
\frac{2}{0}\end{array}$ & 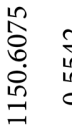 & $\begin{array}{c}\text { F̂ } \\
\text { on } \\
\text { in } \\
0\end{array}$ & & 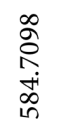 & $\sqrt{-7}$ & $\begin{array}{l}\overrightarrow{\vec{\sigma}} \\
\stackrel{0}{\dot{P}} \\
\vec{q}\end{array}$ & fi \\
\hline$\Leftrightarrow$ & 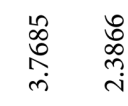 & 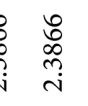 & 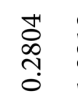 & 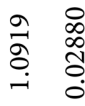 & $\begin{array}{l}\text { ते } \\
\text { ลे }\end{array}$ & 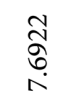 & $\stackrel{\stackrel{M}{n}}{\stackrel{\Xi}{=}}$ & $\begin{array}{l}\hat{0} \\
6 \\
i \\
i\end{array}$ & $\begin{array}{l}\infty \\
\stackrel{0}{0} \\
\stackrel{-}{0}\end{array}$ & 号 & $\begin{array}{l}\stackrel{\partial}{\partial े} \\
\stackrel{\partial}{0}\end{array}$ & 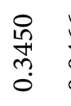 & \begin{tabular}{l}
$\stackrel{0}{\circ}$ \\
\multirow{1}{0}{} \\
0
\end{tabular} & $\begin{array}{l}\vec{m} \\
\overrightarrow{2} \\
0\end{array}$ & 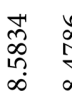 & \begin{tabular}{l}
$\stackrel{8}{\infty}$ \\
\multirow{4}{+}{} \\
$\infty$
\end{tabular} & & 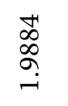 & & \begin{tabular}{l}
\multirow{7}{7}{} \\
10 \\
0
\end{tabular} & \\
\hline u & 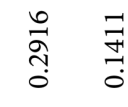 & $\begin{array}{l}\vec{\exists} \\
\overrightarrow{0}\end{array}$ & $\underset{\substack{n \\
0 \\
0}}{0}$ & 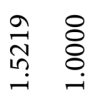 & $\begin{array}{l}\hat{A} \\
\hat{0} \\
0\end{array}$ & $\begin{array}{l}\hat{\sigma} \\
0 \\
0 \\
0\end{array}$ & $\begin{array}{l}\stackrel{n}{ } \\
\text { hn } \\
\text { in }\end{array}$ & $\begin{array}{l}\widetilde{1} \\
\infty \\
\tilde{0} \\
0\end{array}$ & $\begin{array}{l}8 \\
0 \\
\infty \\
0 \\
0\end{array}$ & $\begin{array}{l}\circ \\
\stackrel{0}{0} \\
\stackrel{0}{0}\end{array}$ & $\begin{array}{l}\infty \\
\substack{\infty \\
\infty \\
\infty \\
0}\end{array}$ & $\begin{array}{l}\stackrel{0}{0} \\
\stackrel{0}{0} \\
\stackrel{0}{0}\end{array}$ & 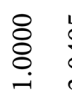 & $\begin{array}{l}\stackrel{n}{2} \\
\stackrel{+}{+} \\
\dot{m}\end{array}$ & 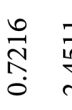 & ت্ & & 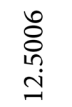 & & $\underset{+}{\stackrel{ }{\sim}}$ & $\vec{i}$ \\
\hline 0 & 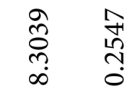 & 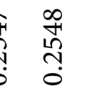 & 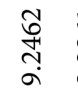 & 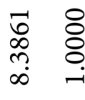 & $\begin{array}{l}a \\
0 \\
0 \\
0 \\
0 \\
0\end{array}$ & $\begin{array}{l}0 \\
0 \\
\stackrel{0}{0} \\
0\end{array}$ & $\begin{array}{l}\stackrel{8}{0} \\
\stackrel{0}{0} \\
0\end{array}$ & 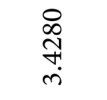 & 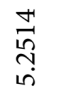 & 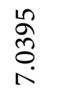 & 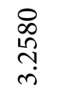 & 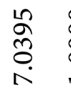 & 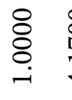 & 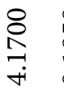 & $\begin{array}{ll}0 & \\
\infty & \\
\infty & 0 \\
0 & 0 \\
0 & 0\end{array}$ & $\begin{array}{l}\stackrel{0}{1} \\
\text { !ी } \\
0 \\
0\end{array}$ & & $\begin{array}{l}\vec{\infty} \\
0 \\
0 \\
\stackrel{0}{\oplus}\end{array}$ & & 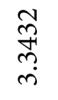 & ن. \\
\hline$\theta$ & 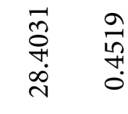 & 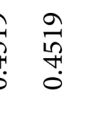 & $\begin{array}{l}\stackrel{n}{0} \\
\stackrel{+}{\infty} \\
\infty \\
\dot{n}\end{array}$ & 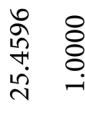 & 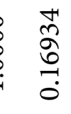 & $\begin{array}{l}\text { से } \\
\text { Oे } \\
0 \\
0\end{array}$ & $\begin{array}{l}\hat{o} \\
\text { oे } \\
\dot{r}\end{array}$ & 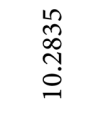 & $\begin{array}{l}\vec{\infty} \\
\infty \\
\stackrel{0}{0}\end{array}$ & $\underset{\substack{0\\
}}{\stackrel{n}{n}}$ & 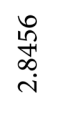 & $\begin{array}{l}\stackrel{0}{0} \\
\stackrel{1}{n}\end{array}$ & 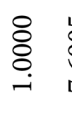 & \begin{tabular}{l}
$n$ \\
\multirow{2}{0}{} \\
مे
\end{tabular} & 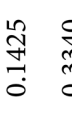 & $\begin{array}{l}\text { O+1 } \\
\text { m. } \\
\text { ?. } \\
0\end{array}$ & & $\begin{array}{l}\stackrel{n}{1} \\
\stackrel{m}{0} \\
0\end{array}$ & & 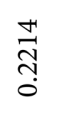 & t. \\
\hline & 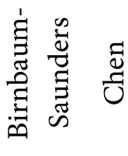 & 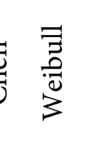 & 山 & 苐 & ప్ & 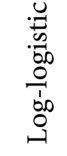 & 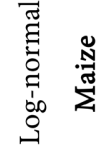 & 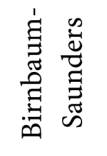 & ฮ্ & $\begin{array}{l}\bar{Z} \\
\frac{\overline{0}}{\overline{0}} \\
z\end{array}$ & 山 & 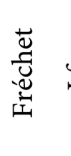 & $\stackrel{\leftrightarrows}{\leftrightarrows}$ & ق. & 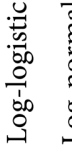 & . & 悹 & $\infty$ & & $\overline{\bar{z}}$ & \\
\hline
\end{tabular}




\begin{tabular}{|c|c|c|c|c|c|c|c|c|c|c|c|c|c|c|c|c|c|c|c|c|c|c|c|}
\hline 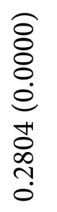 & 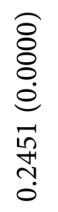 & 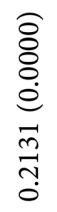 & 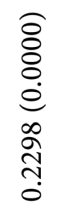 & 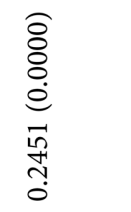 & 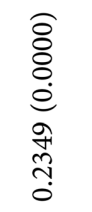 & 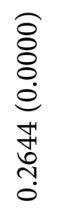 & 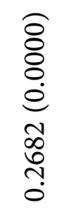 & 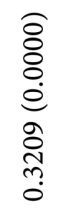 & 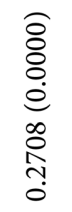 & 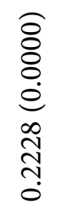 & 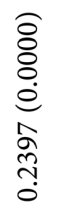 & 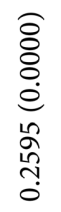 & 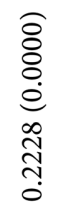 & & 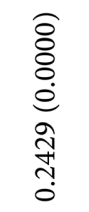 & 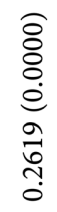 & 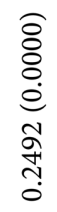 & $\begin{array}{l}\widehat{ᄋ} \\
\delta \\
0 \\
\stackrel{0}{0} \\
\vec{m} \\
\stackrel{\oplus}{0} \\
0\end{array}$ & 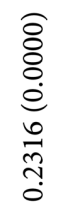 & 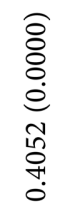 & 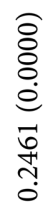 & & 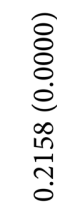 \\
\hline 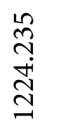 & 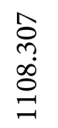 & $\begin{array}{l}\stackrel{n}{\infty} \\
\stackrel{\infty}{0} \\
\stackrel{\infty}{=}\end{array}$ & 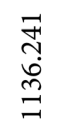 & 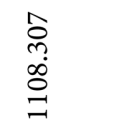 & 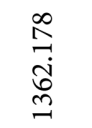 & 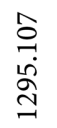 & 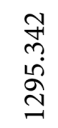 & $\begin{array}{l}\hat{b} \\
0 \\
0 \\
0 \\
-0\end{array}$ & 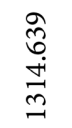 & 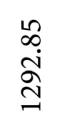 & \begin{tabular}{l}
$\mathbb{H}$ \\
\multirow{n}{n}{} \\
0 \\
$\vec{m}$
\end{tabular} & 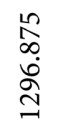 & 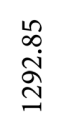 & & 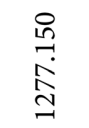 & 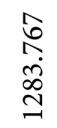 & 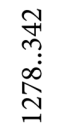 & 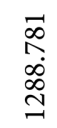 & 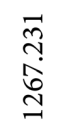 & 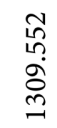 & 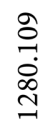 & & 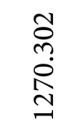 \\
\hline 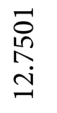 & \begin{tabular}{l}
8 \\
\multirow{0}{1}{} \\
$\infty$ \\
$\infty$ \\
$\infty$
\end{tabular} & $\begin{array}{l}0 \\
\text { \& } \\
0 \\
0 \\
= \\
=\end{array}$ & 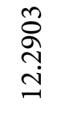 & 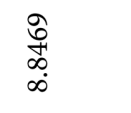 & 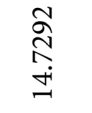 & $\begin{array}{l}\stackrel{0}{1} \\
\stackrel{\overbrace{}}{G} \\
=\end{array}$ & $\begin{array}{l}\overrightarrow{\tilde{J}} \\
\text { }\end{array}$ & $\begin{array}{l}\tilde{O} \\
\infty \\
\infty \\
\tilde{N}\end{array}$ & $\begin{array}{l}\sigma \\
\hat{\delta} \\
\infty \\
\sigma\end{array}$ & $\underset{\infty}{\stackrel{\infty}{\stackrel{\infty}{人}}}$ & $\begin{array}{l}\bar{\sigma} \\
\vec{a}\end{array}$ & $\begin{array}{l}\text { I } \\
\text { बे } \\
\infty\end{array}$ & $\underset{\infty}{\stackrel{\infty}{\stackrel{N}{人}}}$ & & \begin{tabular}{l}
$\bar{ন}$ \\
\multirow{7}{*}{} \\
$\stackrel{2}{n}$
\end{tabular} & 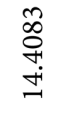 & 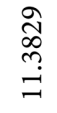 & $\begin{array}{l}\infty \\
\widetilde{N} \\
\tilde{n} \\
0 \\
0\end{array}$ & $\begin{array}{l}\infty \\
\infty \\
i n n \\
i \\
\dot{n}\end{array}$ & 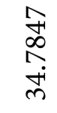 & 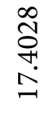 & & $\begin{array}{l}\stackrel{1}{0} \\
\infty \\
0 \\
0\end{array}$ \\
\hline $\begin{array}{l}\sqrt{0} \\
\stackrel{b}{b} \\
\stackrel{i}{i}\end{array}$ & 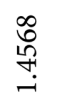 & 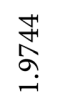 & $\begin{array}{l}\stackrel{H}{2} \\
\stackrel{\text { సे }}{\mathrm{N}}\end{array}$ & 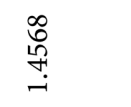 & 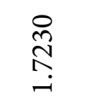 & $\begin{array}{l}\infty \\
\text { Oे } \\
\text { 웃 } \\
i\end{array}$ & 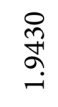 & $\begin{array}{l}\stackrel{2}{\infty} \\
\stackrel{\infty}{\sim} \\
+\end{array}$ & 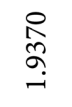 & 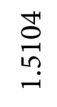 & $\begin{array}{l}\vec{\infty} \\
\stackrel{0}{0} \\
\stackrel{\leftrightarrow}{-}\end{array}$ & $\begin{array}{l}\hat{\overbrace{}} \\
\stackrel{2}{\leftrightarrows}\end{array}$ & 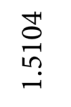 & & 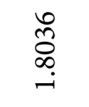 & 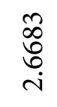 & $\begin{array}{l}\infty \\
\stackrel{0}{0} \\
\stackrel{0}{i} \\
i\end{array}$ & $\underset{\stackrel{\overbrace{}}{\sim}}{\stackrel{\overbrace{}}{\sim}}$ & $\begin{array}{l}\underset{0}{0} \\
\stackrel{\sim}{\sim}\end{array}$ & 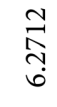 & $\begin{array}{l}\text { oे } \\
\text { గ్ } \\
-i\end{array}$ & $\stackrel{\infty}{\stackrel{\infty}{\sigma}}$ & $\begin{array}{l}\infty \\
\infty \\
i n \\
i n\end{array}$ \\
\hline 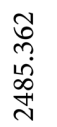 & 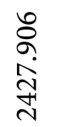 & 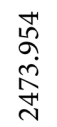 & 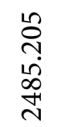 & 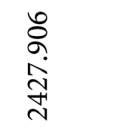 & $\begin{array}{l}\text { na } \\
\stackrel{\leftrightarrow}{\dot{+}} \\
\stackrel{+}{m}\end{array}$ & 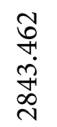 & 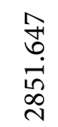 & 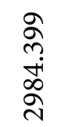 & 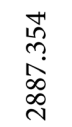 & $\begin{array}{l}\text { मे } \\
0 \\
0 \\
\infty \\
\infty \\
\infty \\
\text { N }\end{array}$ & $\begin{array}{l}\stackrel{\infty}{0} \\
\stackrel{\infty}{\infty} \\
\stackrel{\infty}{\infty} \\
\stackrel{\sim}{N}\end{array}$ & 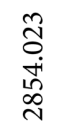 & 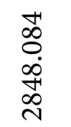 & & 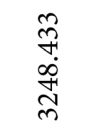 & 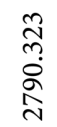 & 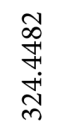 & 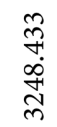 & 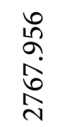 & $\begin{array}{l}\text { मे } \\
\text { مी } \\
\text { N } \\
\infty \\
\infty \\
N\end{array}$ & $\begin{array}{l}\text { م } \\
\underset{+}{+} \\
\stackrel{\sim}{\sim}\end{array}$ & & 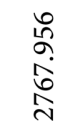 \\
\hline 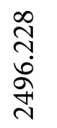 & $\begin{array}{l}\stackrel{M}{N} \\
\infty \\
\infty \\
\stackrel{\infty}{\sim}\end{array}$ & 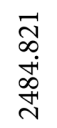 & 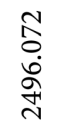 & 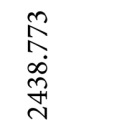 & $\begin{array}{l}\text { 今゙ } \\
\text { in } \\
\stackrel{1}{m}\end{array}$ & 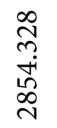 & 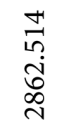 & $\begin{array}{l}\hat{0} \\
\text { ஸू } \\
\text { ลे }\end{array}$ & $\begin{array}{l}\overrightarrow{\text { ते }} \\
\infty \\
\infty \\
\infty \\
\infty\end{array}$ & $\begin{array}{l}\vec{n} \\
\alpha \\
\infty \\
\infty \\
\infty \\
\infty\end{array}$ & 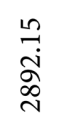 & $\begin{array}{l}a \\
\infty \\
\infty \\
+\stackrel{0}{0} \\
\stackrel{\sim}{N}\end{array}$ & $\begin{array}{l}\vec{n} \\
\alpha \\
\infty \\
\infty \\
\infty \\
\infty\end{array}$ & & 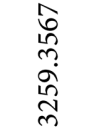 & 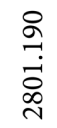 & 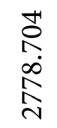 & 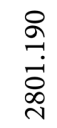 & 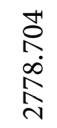 & 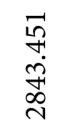 & 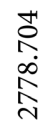 & & $\begin{array}{c}\widetilde{0} \\
\infty \\
\infty \\
\infty\end{array}$ \\
\hline 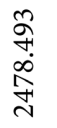 & 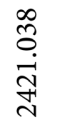 & 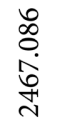 & 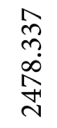 & $\begin{array}{l}\infty \\
\stackrel{\infty}{0} \\
\stackrel{\vec{H}}{\sim}\end{array}$ & $\begin{array}{l}\stackrel{n}{0} \\
\stackrel{m}{m} \\
\stackrel{m}{m}\end{array}$ & 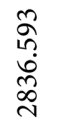 & 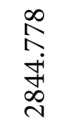 & $\begin{array}{l}\hat{n} \\
\stackrel{n}{\hat{N}} \\
\hat{\text { N }}\end{array}$ & 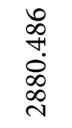 & $\begin{array}{l}\stackrel{0}{\sim} \\
\stackrel{\sim}{*} \\
\stackrel{\sim}{\sim}\end{array}$ & $\begin{array}{l}\underset{+}{J} \\
\underset{+}{+} \\
\stackrel{\infty}{\sim}\end{array}$ & 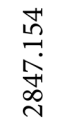 & 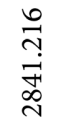 & & 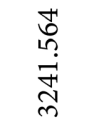 & 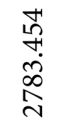 & 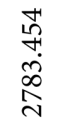 & $\begin{array}{l}\text { oे } \\
\text { : } \\
\stackrel{0}{\circ}\end{array}$ & 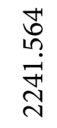 & 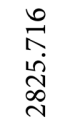 & $\begin{array}{l}\text { ठे } \\
\text { : } \\
\stackrel{0}{0} \\
\text { N }\end{array}$ & & $\begin{array}{l}\infty \\
\stackrel{0}{0} \\
\stackrel{\vec{\nabla}}{\Delta} \\
\stackrel{\sim}{\sim}\end{array}$ \\
\hline 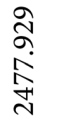 & 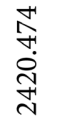 & 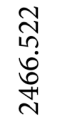 & 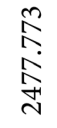 & $\begin{array}{l}\text { H } \\
\text { H. } \\
\stackrel{\mathbb{H}}{\mathrm{H}}\end{array}$ & $\begin{array}{l}\vec{F} \\
\text { i } \\
\stackrel{m}{m} \\
m\end{array}$ & $\begin{array}{l}\text { ठे. } \\
\dot{0} \\
\infty \\
\text { ஸे }\end{array}$ & $\begin{array}{l}\underset{+}{N} \\
\underset{+}{+} \\
\underset{N}{N}\end{array}$ & 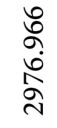 & 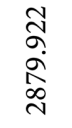 & 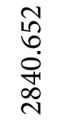 & 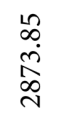 & 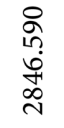 & 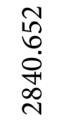 & & $\begin{array}{l}\vec{\delta} \\
\dot{\sim} \\
\vec{\sim}\end{array}$ & 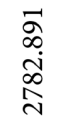 & 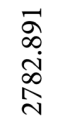 & 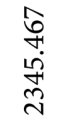 & $\begin{array}{l}n \\
\stackrel{o}{+} \\
0 \\
0 \\
\stackrel{0}{N}\end{array}$ & 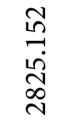 & 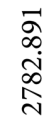 & & 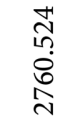 \\
\hline 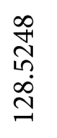 & 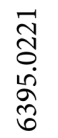 & 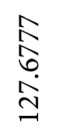 & 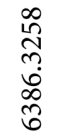 & 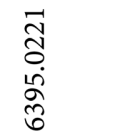 & 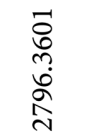 & 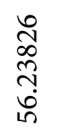 & 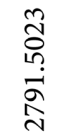 & 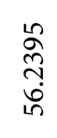 & $\begin{array}{l}\vec{D} \\
\stackrel{+}{+} \\
\dot{\widehat{N}}\end{array}$ & 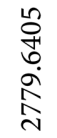 & $\begin{array}{l}\text { त् } \\
\infty \\
\infty \\
\dot{n} \\
\dot{n}\end{array}$ & 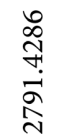 & 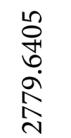 & & 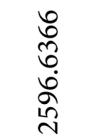 & 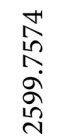 & 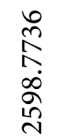 & 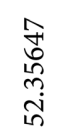 & 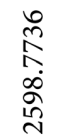 & $\stackrel{n}{\bar{\Xi}}$ & 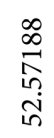 & & $\begin{array}{l}\stackrel{\circ}{0} \\
\stackrel{0}{0} \\
\stackrel{8}{\Delta}\end{array}$ \\
\hline $\begin{array}{l}\infty \\
0 \\
0 \\
0 \\
0 \\
\\
\end{array}$ & 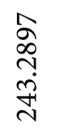 & ڤั & $\begin{array}{l}\hat{\hat{\sigma}} \\
\text { ळे } \\
\text { in }\end{array}$ & 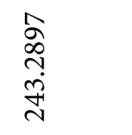 & 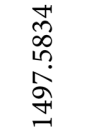 & 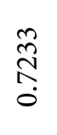 & 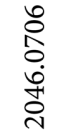 & $\begin{array}{l}\stackrel{\circ}{\circ} \\
\stackrel{\circ}{+} \\
+\end{array}$ & $\begin{array}{l}8 \\
0 \\
\infty \\
\dot{\infty} \\
0 \\
0\end{array}$ & 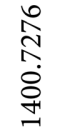 & 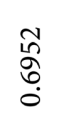 & 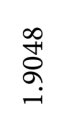 & 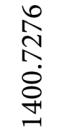 & & 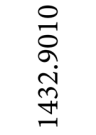 & $\begin{array}{l}\text { Rิ } \\
\text { స్ }\end{array}$ & 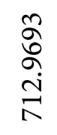 & 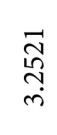 & 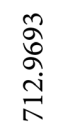 & $\stackrel{2}{8}$ & 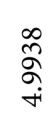 & $\begin{array}{l}\text { గn } \\
\text { ถุ }\end{array}$ & $\begin{array}{l}\text { నิ } \\
\text { న. } \\
\text { న⿱ }\end{array}$ \\
\hline बे & 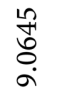 & 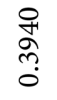 & 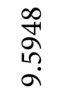 & 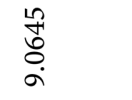 & 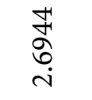 & $\begin{array}{l}\stackrel{H}{H} \\
0 \\
0 \\
0 \\
0\end{array}$ & 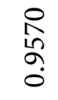 & 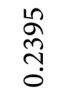 & $\begin{array}{l}\text { Oे } \\
0 \\
\because \\
0\end{array}$ & $\begin{array}{l}\hat{o} \\
\stackrel{0}{0} \\
\stackrel{h}{h}\end{array}$ & $\begin{array}{l}\stackrel{n}{\hat{O}} \\
\stackrel{-}{0} \\
0\end{array}$ & 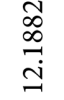 & $\begin{array}{l}\hat{o} \\
\stackrel{0}{0} \\
\stackrel{h}{n}\end{array}$ & & $\begin{array}{l}\text { مे } \\
\text { b. } \\
\text { in }\end{array}$ & $\begin{array}{l}\overrightarrow{0} \\
\stackrel{0}{0} \\
0 \\
0\end{array}$ & $\begin{array}{l}\vec{\partial} \\
\stackrel{\Delta}{\infty} \\
i\end{array}$ & 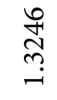 & $\begin{array}{l}\vec{a} \\
\stackrel{1}{\alpha} \\
i \\
i\end{array}$ & $\begin{array}{l}\text { N̂} \\
\text { ty } \\
\text { O. }\end{array}$ & 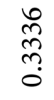 & $\begin{array}{l}\text { సิ } \\
\text { గ్ర }\end{array}$ & : \\
\hline 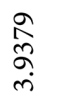 & 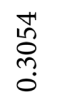 & 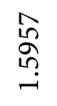 & $\begin{array}{l}\stackrel{n}{0} \\
\stackrel{\circ}{n} \\
i n\end{array}$ & $\begin{array}{l}\text { H } \\
\text { 号 } \\
\text { ஸे } \\
0\end{array}$ & $\begin{array}{l}\stackrel{2}{7} \\
\stackrel{+}{0}\end{array}$ & $\begin{array}{l}\stackrel{\infty}{-} \\
\stackrel{0}{-}\end{array}$ & $\begin{array}{l}\hat{O} \\
\text { ర్ } \\
\dot{0}\end{array}$ & 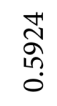 & 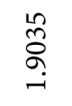 & $\begin{array}{l}\infty \\
\infty \\
0 \\
0 \\
0 \\
0\end{array}$ & $\begin{array}{l}\underset{\sim}{\mathbb{O}} \\
\stackrel{\infty}{-}\end{array}$ & $\begin{array}{l}8 \\
\stackrel{0}{\circ} \\
\stackrel{+}{\infty}\end{array}$ & $\begin{array}{l}\infty \\
\infty \\
: 0 \\
0 \\
0\end{array}$ & & 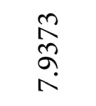 & $\begin{array}{l}\tilde{n} \\
\stackrel{2}{0}+1 \\
0 \\
0\end{array}$ & 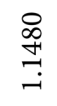 & $\begin{array}{l}\hat{o} \\
\text { ֵू } \\
\delta\end{array}$ & 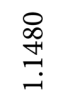 & ڤ̊̀ & 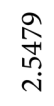 & $\begin{array}{l}\stackrel{\infty}{\infty} \\
\stackrel{\sim}{\sim}\end{array}$ & $\begin{array}{l}\stackrel{2}{c} \\
\text { in } \\
\text { in }\end{array}$ \\
\hline $\begin{array}{l}\stackrel{\infty}{\infty} \\
\stackrel{\infty}{0} \\
\stackrel{+}{+}\end{array}$ & $\begin{array}{l}\qquad 0 \\
\stackrel{\circ}{0} \\
0\end{array}$ & $\begin{array}{l}m \\
\stackrel{0}{0} \\
\stackrel{0}{0} \\
\stackrel{0}{0}\end{array}$ & $\begin{array}{l}\hat{\Omega} \\
\hat{\Omega} \\
\stackrel{n}{n}\end{array}$ & $\begin{array}{l}n \\
\stackrel{1}{\circ} \\
\stackrel{7}{0}\end{array}$ & $\begin{array}{l}\overrightarrow{\overparen{F}} \\
\text { i }\end{array}$ & $\stackrel{\text { ڤે }}{\stackrel{\overbrace{}}{-}}$ & $\begin{array}{l}0 \\
\infty \\
\infty \\
\infty \\
0\end{array}$ & $\begin{array}{l}\text { ते } \\
\text { 官 } \\
\dot{m}\end{array}$ & $\begin{array}{l}\hat{\infty} \\
\infty \\
\stackrel{0}{0} \\
0\end{array}$ & $\begin{array}{l}\text { 암 } \\
\text { 웅 } \\
0\end{array}$ & 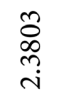 & $\begin{array}{l}\text { O̊̊. } \\
\stackrel{f}{0} \\
0\end{array}$ & $\begin{array}{l}\text { 웅 } \\
\text { - } \\
0 \\
0\end{array}$ & & $\begin{array}{l}\overrightarrow{7} \\
\text { in } \\
\infty\end{array}$ & 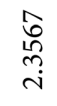 & $\begin{array}{l}\widetilde{N} \\
\stackrel{乛}{0}\end{array}$ & $\begin{array}{l}\tilde{N} \\
\hat{\widehat{O}} \\
\text { ì }\end{array}$ & 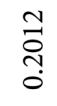 & 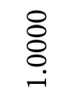 & $\begin{array}{l}\frac{1}{\alpha} \\
\infty \\
\infty \\
0\end{array}$ & ڤి & $\begin{array}{l}\infty \\
\hat{\infty} \\
\stackrel{0}{0} \\
\stackrel{+}{+}\end{array}$ \\
\hline 亗 & $\begin{array}{l}\text { ڤે } \\
\text { ஸે }\end{array}$ & 芯 & $\begin{array}{l}\text { f } \\
\text { त̀ } \\
\text { ind }\end{array}$ & $\begin{array}{l}\text { مे̀ } \\
\text { ஸे }\end{array}$ & 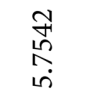 & 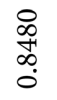 & $\begin{array}{l}\text { 员 } \\
\text { مू } \\
0\end{array}$ & 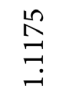 & 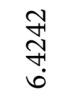 & $\begin{array}{l}\frac{H}{5} \\
\stackrel{7}{0}\end{array}$ & $\begin{array}{l}\stackrel{a}{ } \\
\stackrel{\partial}{+} \\
\stackrel{n}{+}\end{array}$ & $\underset{\vec{m}}{\vec{m}}$ & $\begin{array}{l}\frac{H}{5} \\
\stackrel{7}{0}\end{array}$ & & $\begin{array}{l}\stackrel{0}{1} \\
\text { 彳̀ } \\
\text { o. }\end{array}$ & 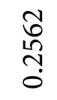 & $\begin{array}{l}\widehat{\infty} \\
\infty \\
\stackrel{0}{0} \\
0\end{array}$ & $\begin{array}{l}\text { م̂} \\
\hat{O} \\
\text { in }\end{array}$ & $\begin{array}{l}\widehat{\infty} \\
\infty \\
0 \\
0 \\
0\end{array}$ & ఫ̊ & $\begin{array}{l}n \\
\tilde{0} \\
\infty \\
0\end{array}$ & $\begin{array}{l}\stackrel{10}{3} \\
\stackrel{0}{0}\end{array}$ & 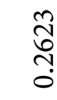 \\
\hline 莺 & $\stackrel{\leftrightarrows}{~}$ & 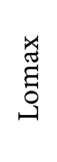 & $\begin{array}{l}\widetilde{J} \\
\stackrel{0}{0} \\
0 \\
0 \\
0 \\
0 \\
0\end{array}$ & 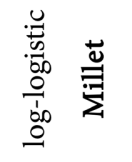 & 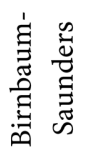 & 莺 & $\begin{array}{l}\overline{3} \\
\overline{0} \\
\overline{3}\end{array}$ & 山 & 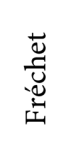 & $\stackrel{\leftrightarrows}{\leftrightarrows}$ & 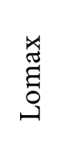 & 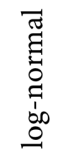 & 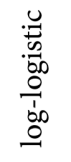 & $\begin{array}{l}5 \\
0 \\
0 \\
\vdots\end{array}$ & 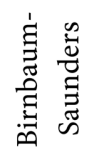 & $\begin{array}{l}\text { चี } \\
\text { चี }\end{array}$ & $\begin{array}{l}\overline{7} \\
\frac{0}{\overline{0}} \\
3\end{array}$ & 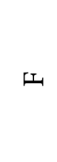 & 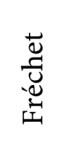 & 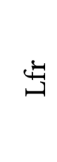 & 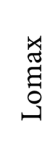 & & 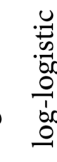 \\
\hline
\end{tabular}




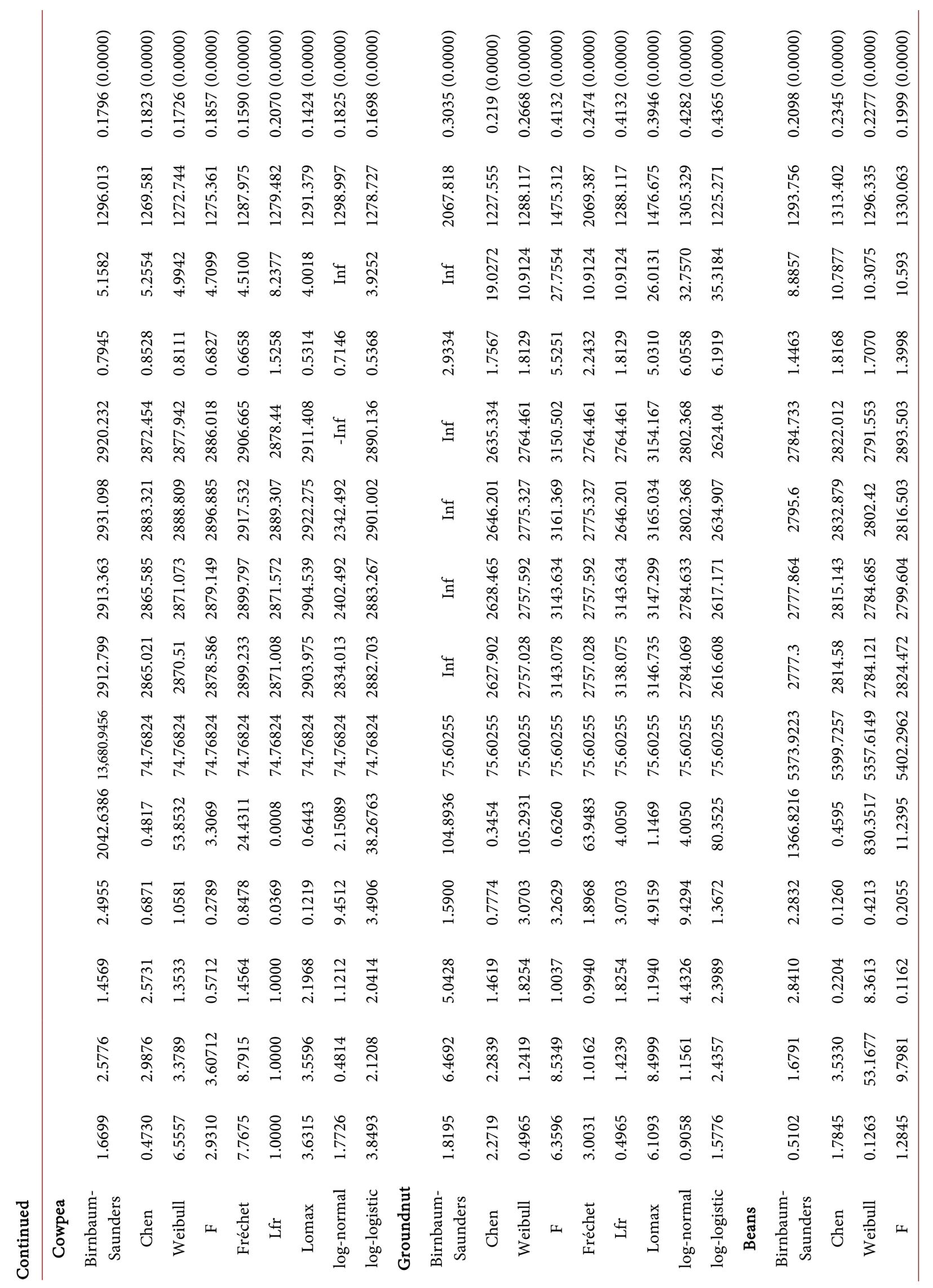




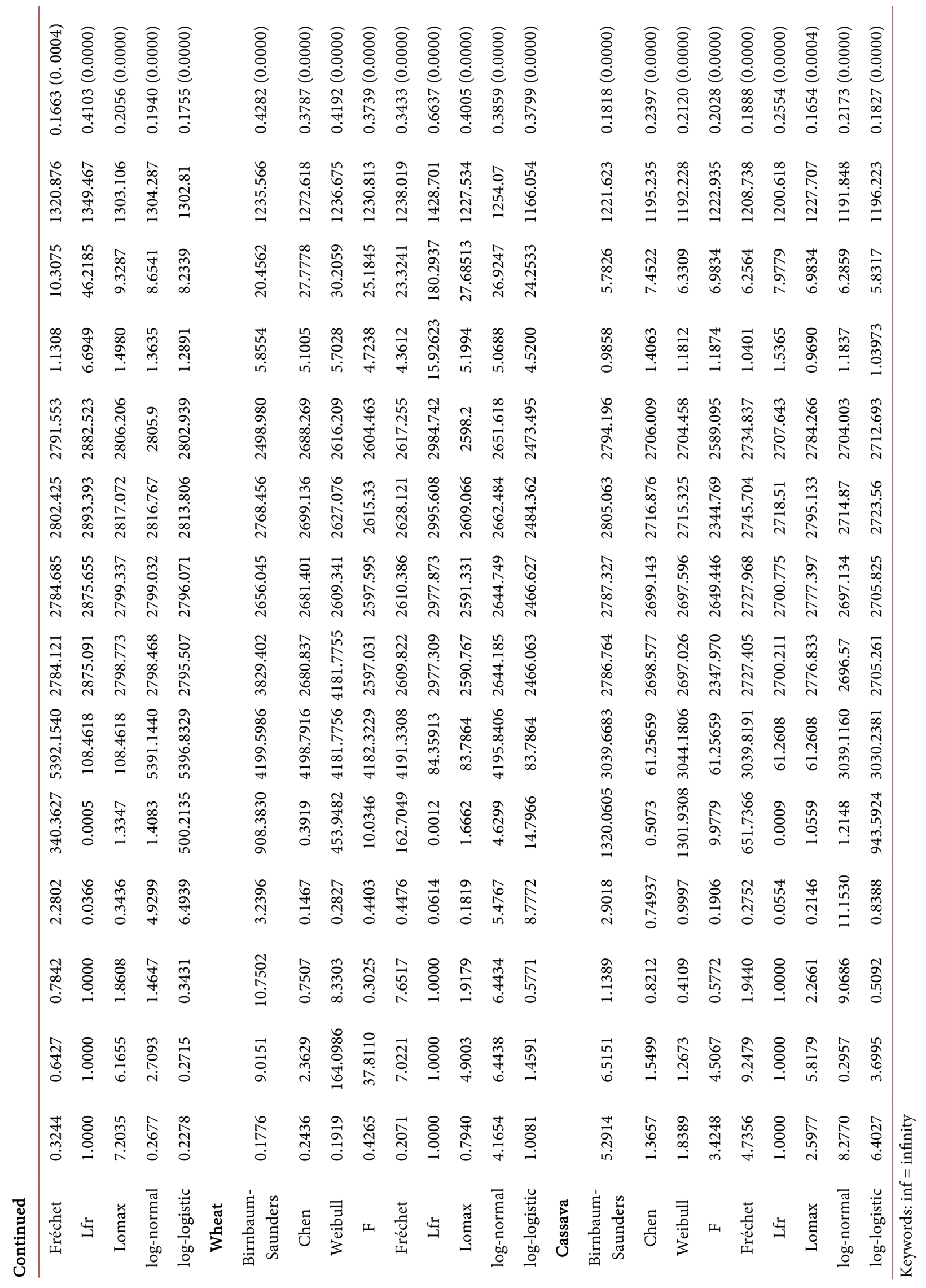


is $15.4291>0.7752$ and Kolomogorov-Smirnovs' p-value $=0.0000<0.05$. Lognormal possessed reduced-error model performance for cowpea with AIC = 2834.013; $\mathrm{CAIC}=2402.492 ; \mathrm{BIC}=2342.492$, where

$\hat{\Theta}=(1.7726,0.4814,1.1212,9.4512,2.15089,74.7648)$ and induced parameter of $\Omega=(9.4512,2.15089,74.7648)$ such that it's Kolomogorov-Smirnovs' $\mathrm{p}$-value $=$ $0.0000<0.05$. Log-logistic random noise produced the ideal performance for price of groundnut with $\hat{\Theta}=(1.5776,2.4357,2.3989,1.3672,80.3525,75.6026)$, induced parameters $\hat{\Omega}=(1.3672,80.3525,75.6026)$. Its Anderson-Darling estimate is $35.3184>0.7752$ and Kolomogorov-Smirnovs' p-value $=0.0000<0.05$.

Birnbaum-Saunders possessed the ideal reduced-error model performance for price of beans with $\mathrm{AIC}=2777.3$; $\mathrm{CAIC}=2777.864 ; \mathrm{BIC}=2795.6$; $\mathrm{HQIC}=$ 2784.733, where $\hat{\Theta}=(0.5102,1.6791,2.8410,2.2832,1366.8216,5373.9233)$, such that $\hat{\Omega}=(2.2832,1366.8216,5373.9223)$. Its Anderson-Darling estimate is $8.8857>0.7752$ and Kolomogorov-Smirnovs' p-value $=0.0000<0.05$. Loglogistic is the paragon random noise for price of wheat with $\mathrm{AIC}=2466.063$; CAIC $=$ 2466.627; $\mathrm{BIC}=2484.362$; HQIC $=2473.495$, where,

$\hat{\Theta}=(1.0081,1.4591,0.5771,8.7772,14.7966,83.7864)$, such that

$\hat{\Omega}=(8.7772,14.7966,83.7864)$. Its Anderson-Darling estimate is

$24.2533>0.7752$ and Kolomogorov-Smirnovs' $\mathrm{p}$-value $=0.0000<0.05$. Lastly, $\mathrm{F}$ surmounted other Generalized Beta-G distributional random noises for price of cassava with $\mathrm{AIC}=2347.970 ; \mathrm{CAIC}=2649.446 ; \mathrm{BIC}=2344.769 ; \mathrm{HQIC}=$ $2589.095 \hat{\Theta}=(3.4248,4.5067,0.5772,0.1906,9.9779,61.2566)$ such that, $\hat{\Omega}=(0.1906,9.9779,61.2566)$. Its Anderson-Darling estimate is $6.9834>0.7752$ and Kolomogorov-Smirnovs' p-value $=0.0000<0.05$.

\section{Conclusion}

In conclusion, Lomax and Linear Failure Rate (LFR) out of the Generalized Beta-G family of distributions are ineffective in modelling the prices of all the cereals studied. This might be due to the fact that LFR is peculiar to survival, censored and uncensored analysis. Aditionally, Lomax distribution (otherwise knwon as Pareto Type II distrbution) might be ineffectual in living-up to expectation due to its peculiarity in statistical modelling of tailedness observations, reliability studies and life testing problems in survival studies. Chen and loglogistic distributional random noises are the leading candidates among the Generalized Beta-G family of distributions in modelling of price returns, followed by Fréchet random noise. Weibull, Birnbaum-Saunders, and $\mathrm{F}$ distributional random noises gave un-recommendable higher error performances. Overall, all the distributional random noises for the Generalized Beta-G family of distributions gave Kolmogorov-Smirnov's p-values that are far lesser than 0.05 and Anderson-Darling estimates that are greater than the critical value of 0.7752 to affirm the model adequacy of the Generalized Beta-G family of distributions, in contrast to unbounded distributions. Among the limitations of Beta-G family of distributions is that it is for positive continuous values with single mode, and its 1 to many mapping car- 
rier is based on either $[0,1]$ or $(0,1)$.

\section{Acknowledgements}

I personally extended gratitude to the Ministry of Agriculture and Natural Resources (MANR), Kano state, Nigeria for the provision of the prices of cereals dataset used in this project.

\section{Conflicts of Interest}

The author declares no conflicts of interest regarding the publication of this paper.

\section{References}

[1] Okasha, H.M., El-Baz, A.H., Tarabia, A.M.K. and Basheer, A.M. (2017) Extended Inverse Weibull Distribution with Reliability Application. Journal of the Egyptian Mathematical Society, 25, 343-349. https://doi.org/10.1016/j.joems.2017.02.006

[2] Barreto-Souza, W., Cordeiro, G.M. and Simas, A.B. (2008) Some Results for Beta Fréchet Distribution.

[3] Teimouri, M. and Nadarajah, S. (2019) Package "MPS": Estimating through the Maximum Product Spacing Approach.

[4] Alzaatreh, A., Lee, C. and Famoye, F. (2013) A New Method for Generating Families of Continuous Distributions. Metron, 71, 63-79. https://doi.org/10.1007/s40300-013-0007-y

[5] Eugene, N., Lee, C. and Famoye, F. (2002) Beta-Normal Distribution and Its Applications. Communications in Statistics-Theory and Methods, 31, 497-512. https://doi.org/10.1081/STA-120003130

[6] Alexander, C., Cordeiro, G.M., Ortega, E.M.M. and Sarabia, J.M. (2012) Generalized Beta-Generated Distributions. Computational Statistics \& Data Analysis, 56, 1880 1897. https://doi.org/10.1016/j.csda.2011.11.015

[7] Ristić, M.M. and Nadarajah, S. (2013) A New Lifetime Distribution. Journal of Statistical Computation and Simulation, 84, 135-150.

https://doi.org/10.1080/00949655.2012.697163

[8] Mudholkar, G.S., Kollia, G.D., Lin, C.T. and Patel, K.R. (1991) A Graphical Procedure for Comparing Goodness-of-Fit Tests. Journal of Royal Statistical Society B, 53, 221-232. https://doi.org/10.1111/j.2517-6161.1991.tb01820.x

[9] Lemonte, A.J., Barreto-Souza, W. and Cordeiro, G.M. (2013) The Exponentiated Kumaraswamy Distribution \& Its Log-Transform. Brazilian Journal of Probability and Statistics, 27, 31-53. https://doi.org/10.1214/11-BJPS149

[10] Ristić, M.M. and Balakrishnan, N. (2012) The Gamma Exponentiated Exponential Distribution. Journal of Statistical Computation and Simulation, 82, 1191-1206.

https://doi.org/10.1080/00949655.2011.574633

[11] Merovci, F., Alizadeh, M., Yousof, H.M. and Hamedani, G.G. (2017) The Exponentiated Transmuted-G Family of Distributions. Theory and Applications Communications in Statistics - Theory and Methods, 46, 10800-10822. https://doi.org/10.1080/03610926.2016.1248782

[12] Gauss, M.C., Alizadeh, M., Ozel, G., Hosseini, B., Ortega, E.M.M. and Altunc, E. (2017) The Generalized Odd Log-Logistic Family of Distributions: Properties, Re- 
gression Models and Applications. Journal of Statistical Computation and Simulation, 87, 908-932. https://doi.org/10.1080/00949655.2016.1238088

[13] Nofal, Z.M., Afify, A.Z., Yousof, H.M. and Cordeiro, G.M. (2016) The Generalized Transmuted-G Family of Distributions. Communications in Statistics-Theory and Methods, 46, 4119-4136. https://doi.org/10.1080/03610926.2015.1078478

[14] Lemonte, A.J. (2013) A New Extension of the Birnbaum-Saunders Distribution. Brazilian Journal of Probability and Statistics, 27, 133-149. https://doi.org/10.1214/11-BJPS160

[15] Gomes, A.E., Silva, C.Q., Cordeiro, G.M. and Ortega, E.M.M. (2013) The Beta Burr III Model for Lifetime Data. Brazilian Journal of Probability and Statistics, 27, $502-$ 543. https://doi.org/10.1214/11-BJPS179

[16] Nadarajah, S. and Kotz, S. (2004) The Beta Gumbel Distribution. Mathematical Problems in Engineering, 10, 323-332. https://doi.org/10.1155/S1024123X04403068

[17] Barreto-Souza, W., Cordeiro, G.M. and Simas, A.B. (2011) Some Results for Beta Fréchet Distribution Communications in Statistics Theory and Methods, 40, 798811. https://doi.org/10.1080/03610920903366149

[18] Smith, R.L. and Naylor, J. (1987) A Comparison of Maximum Likelihood and Bayesian Estimators for the Three-Parameter Weibull Distribution. Applied Statistics, 36, 358-369. https://doi.org/10.2307/2347795

[19] Nadarajah, S., Nassiri, V. and Mohammadpour, A. (2014) Truncated-Exponential Skew Symmetric Distributions. Statistics, 48, 872-886.

https://doi.org/10.1080/02331888.2013.821474

[20] Teimouri, M. (2018) MPS: An R Package for Modelling New Families of Distributions. 